\title{
Effect of nonlinear electrostatic forces on the dynamic behaviour of a capacitive ring based Coriolis Vibrating Gyroscope under severe shock
}

\author{
B. Chouvion ${ }^{\mathrm{a}, *}$, S. McWilliam ${ }^{\mathrm{b}}$, A.A. Popov ${ }^{\mathrm{b}}$ \\ ${ }^{a}$ Ecole Centrale de Lyon, LTDS, CNRS UMR 5513, 69130 Ecully, France \\ ${ }^{b}$ Faculty of Engineering, University of Nottingham, University Park, Nottingham NG7 2RD, UK
}

\begin{abstract}
This paper investigates the dynamic behaviour of capacitive ring-based Coriolis Vibrating Gyroscopes (CVGs) under severe shock conditions. A general analytical model is developed for a multi-supported ring resonator by describing the in-plane ring response as a finite sum of modes of a perfect ring and the electrostatic force as a Taylor series expansion. It is shown that the number of supports can induce mode coupling and that mode coupling occurs when the shock is severe and the electrostatic forces are nonlinear. The influence of electrostatic nonlinearity is investigated by numerically simulating the governing equations of motion. For the severe shock cases investigated, when the electrode gap reduces by $\sim 60 \%$, it is found that three ring modes of vibration $(1 \theta, 2 \theta$ and $3 \theta)$ and a $9^{\text {th }}$ order force expansion are needed to obtain converged results for the global shock behaviour. Numerical results when the $2 \theta$ mode is driven at resonance indicate that electrostatic nonlinearity introduces mode coupling which has potential to reduce sensor performance under operating conditions. Under some circumstances it is also found that severe shocks can cause the vibrating response to jump to another stable state with much lower vibration amplitude. This behaviour is mainly a function of shock amplitude and rigid-body motion damping.
\end{abstract}

Keywords: Vibrating ring gyroscope, electrostatic forcing, shock response, nonlinear dynamics

\section{Introduction}

Coriolis Vibrating Gyroscopic sensors (CVGs) are used to measure angular velocity (rate) of a body about a particular axis based on the harmonic vibration response of a degenerate resonator subjected to Coriolis forces. Micro-engineered CVGs are 5 used increasingly in inertial guidance applications due to their small size and low cost, and on-going research is focused on improving the accuracy of these Micro-ElectroMechanical Systems (MEMS) for high performance applications. CVGs are required

\footnotetext{
${ }^{*}$ Corresponding author. Tel.: +33 472186767.

Email address: benjamin. chouvion.ec-lyon.fr (B. Chouvion)
} 
to operate in increasingly harsh environmental conditions [1] and it is important to ensure external shock inputs do not affect the accuracy of rate measurements made.

10 For resonators operating in the linear regime, sensors based on axi-symmetric resonators, such as rings and slotted discs [2], are advantageous because the in-plane flexural modes of vibration occur in degenerate pairs and a shock input does not induce any coupling between modes [3]. However, under severe shock inputs the resonator response is nonlinear and this advantage is lost. In practice, numerous sources of me-

15 chanical nonlinearity are present in resonators, but the dominant source of nonlinearity in state-of-the-art capacitive MEMS sensors is caused by electrostatic forces used to drive and sense the response of the resonator. Under normal operating conditions the resonator vibrates with small amplitude causing the electrode gap size to vary by a small amount and ensuring the system operates within the linear regime. However, under severe shock conditions the electrode gap size can vary significantly due to large amplitude in-plane rigid body motions of the resonator, causing the electrostatic forces to vary nonlinearly.

The aim of this paper is to develop and apply an analytical model to quantify and understand the effect of nonlinear electrostatic forces on the dynamic response and 25 mode coupling in capacitive ring based CVGs under severe shock conditions. Previous work $[4,5]$ has investigated the effect of nonlinear electrostatic forces on mode coupling under pure shock conditions. The model used to achieve this involved expressing the ring response in terms of the modes for an unsupported ring and expanding the electrostatic force as a Taylor series. This approach is generalised here and used to

30 investigate the influence of severe shocks when the ring is driven at resonance under normal operating conditions. The simulation results obtained demonstrate the presence of mode coupling under severe shock conditions and suggest the possibility of jump phenomenon when the ring is driven hard under particular conditions of shock amplitude and damping, which both have potential to diminish sensor performance

35 Thentarily.

The paper is structured as follows. Section 2 develops a model to describe the vibration response of the ring resonator taking into account nonlinear electrostatic forces, including the presence of supports uniformly spaced around the ring circumference. This model generalises the model presented in [4] to any number of supports, investi40 gates the influence of the number of support legs on mode coupling, and confirms the so-called frequency splitting rules $[6,7]$. Section 3 presents a systematic study into the nonlinear response of ring resonators under severe shock conditions for a device recently reported in the literature [1]. The analysis includes convergence studies for the number of expansion terms and modes to accurately model the nonlinear behaviour, time-history and spectrogram results to demonstrate the rich nonlinear dynamics and mode coupling under severe shock conditions, and an investigation into the impact of using inner and outer electrodes. It is anticipated that the model and results presented will guide future development of high performance capacitive CVGs.

\section{Ring resonator modelling}

50

In this section, a general linear mechanical model is developed for a supported ring resonator surrounded by capacitive electrodes subjected to combined harmonic and 
shock excitations.

Different vibrating ring gyroscopes designs are reported in the literature $[1,4,8,9]$. All designs consist of a ring resonator supported by flexible support legs and surrounded by electrodes. The support legs can be fixed to a rigid base via a central hub or externally to the ring, and are uniformly spaced around the ring circumference. Figure 1 shows a schematic diagram of the gyroscope that will be studied in detail in Section 3 in which the vibrating ring is surrounded by electrodes for driving and sensing, and the ring is supported by legs connected to a fixed rigid hub. This device only

60 includes electrodes outside the ring, but the model will have the option to incorporate inner electrodes. In operation the in-plane ring $2 \theta$-mode of the resonator is driven into resonance and the device is subjected to external shock excitations. The resulting motion of the resonator causes the support legs to deform and the radial spacing between the ring and surrounding electrodes to change. The in-plane ring motion is limited by 65 the capacitor gap size, which is much smaller than the ring radial thickness. As the rigid body displacement and elastic deformations of the ring are small, a linear model of the ring and supports is used to describe the ring motion. No attempt is made to model ring-electrode contact.

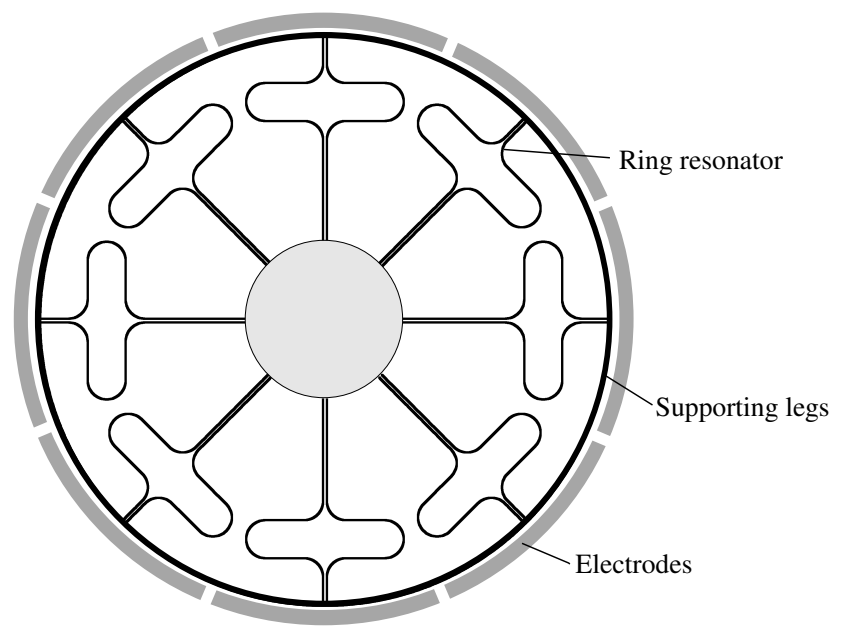

Figure 1: Schematic view of the studied vibrating ring gyroscope [1]

The ring is modelled as a thin, perfect ring having mean radius $r$, radial thickness $h$, axial length $l$, and cross-sectional area $A=h l$. The in-plane ring displacement is expressed as the sum of in-plane rigid body and flexural mode shapes for a perfect ring whose modes occur in degenerate pairs [3]. The support legs connecting the ring and base consist of thin beam (straight or curved) structures, and are modelled as radial and tangential springs for the assumed range of ring deflections. For the resonator shown

75 in Fig. 1, the legs are arranged in pairs and the legs pair can be represented as a single leg having linear radial and tangential springs. The inertia of the legs is modelled as point masses $m_{l}$. The electrostatic forces associated with electrodes located inside and outside the ring are modelled as parallel plate capacitors. The shock excitation is 
modelled as base excitation and the equations of motion for the system are obtained using Lagrange's equation.

In general, the absolute displacement $z(\theta)$ of an element of the ring located at angle $\theta$ can be expressed as:

$$
z(\theta)=z_{b}+\boldsymbol{R} \boldsymbol{u},
$$

where vector $z(\theta)=\left[\begin{array}{ll}x & y\end{array}\right]^{\mathrm{T}}$ represents the absolute displacement of the element, vector $z_{b}=\left[\begin{array}{ll}x_{b} & y_{b}\end{array}\right]^{\mathrm{T}}$ represents the absolute rigid body displacements of the ring centre,

$85 \quad$ vector $\boldsymbol{u}=\left[\begin{array}{ll}w & u\end{array}\right]^{\mathrm{T}}$ represents the flexural displacements of the ring element in radial $(w)$ and tangential $(u)$ directions, and rotation matrix $\mathbf{R}$ resolves the radial and tangential components with the absolute displacement components. Assuming the ring is thin and in-extensible, such that $w=-\partial u / \partial \theta$, and using a Ritz approach, vector $\mathbf{u}$ is expressed in its most general form as:

$$
\boldsymbol{u}(\theta, t)=\boldsymbol{\Psi}^{\mathrm{T}}(\theta) \boldsymbol{\Lambda}(t),
$$

90 where

$$
\boldsymbol{\Psi}^{\mathrm{T}}=\left[\begin{array}{cccccc}
\cdots & \ldots & \cos n \theta & \sin n \theta & \ldots & \ldots \\
\cdots & \cdots & -1 / n \sin n \theta & 1 / n \cos n \theta & \ldots & \ldots
\end{array}\right]
$$

and

$$
\boldsymbol{\Lambda}=\left[\begin{array}{llll}
\cdots & \ldots & {\left[\begin{array}{ll}
q_{n}^{(1)} & q_{n}^{(2)}
\end{array}\right] \quad \ldots \quad \ldots}
\end{array}\right]^{\mathrm{T}},
$$

where $n=1,2, \ldots, N_{R}$ and $q_{n}^{(1)}, q_{n}^{(2)}$ are pairs of generalised coordinates associated with orthogonal shape functions having $n$ nodal diameters - these shape functions are referred to as the $n \theta$-modes. $N_{R}$ defines the number of generalised coordinate pairs 95 used to describe the flexural ring deformation in the Ritz approach, and will be referred to as the Ritz order of approximation in what follows.

\subsection{Kinetic energy}

The kinetic energy of the ring is given by:

$$
T=\frac{1}{2} \int_{V} \rho \dot{z}^{\mathrm{T}} \dot{z} \mathrm{~d} V
$$

where $\rho$ is the material density and $V$ the ring volume.

Using Eqs. (1) to (5) it can be shown that the kinetic energy can be expressed as:

$$
T=\frac{1}{2} \dot{\boldsymbol{h}}^{\mathrm{T}} \boldsymbol{M} \dot{\boldsymbol{h}},
$$


where vector $\boldsymbol{h}$ is equal to $\boldsymbol{h}=\left[\begin{array}{c}\boldsymbol{\Lambda} \\ z_{b}\end{array}\right]$ and mass matrix $\boldsymbol{M}$ is

$$
\boldsymbol{M}=m_{r}\left[\begin{array}{cccccccccc}
1 & 0 & 0 & 0 & \ldots & \ldots & 0 & 0 & 1 & 0 \\
0 & 1 & 0 & 0 & \ldots & \ldots & 0 & 0 & 0 & 1 \\
0 & 0 & 5 / 8 & 0 & \ldots & \ldots & 0 & 0 & 0 & 0 \\
0 & 0 & 0 & 5 / 8 & \ldots & \ldots & 0 & 0 & 0 & 0 \\
\vdots & \vdots & \vdots & \vdots & \ddots & \ddots & \vdots & \vdots & \vdots & \vdots \\
0 & 0 & 0 & 0 & \ldots & \ldots & \frac{N_{R}^{2}+1}{2 N_{R}^{2}} & 0 & 0 & 0 \\
0 & 0 & 0 & 0 & \ldots & \ldots & 0 & \frac{N_{R}^{2}+1}{2 N_{R}^{2}} & 0 & 0 \\
1 & 0 & 0 & 0 & \ldots & \ldots & 0 & 0 & 1 & 0 \\
0 & 1 & 0 & 0 & \ldots & \ldots & 0 & 0 & 0 & 1
\end{array}\right],
$$

where $m_{r}$ is the physical mass of the ring. It is convenient to express the mass matrix in the following compact form:

$$
\boldsymbol{M}=\left[\begin{array}{cc}
\boldsymbol{M}_{\boldsymbol{r}} & \boldsymbol{M}_{\boldsymbol{b r}} \\
\boldsymbol{M}_{\boldsymbol{b r}}{ }^{\mathrm{T}} & m \boldsymbol{I}_{2 \times 2}
\end{array}\right],
$$

where $\boldsymbol{M}_{\boldsymbol{r}}$ is a $2 N_{R} \times 2 N_{R}$ diagonal matrix whose $2 n$ 'th and (2n-1)'th entries are $m_{r}\left(n^{2}+1\right) /\left(2 n^{2}\right)$ i.e. the generalised mass for the $n \theta$-mode and

$$
\boldsymbol{M}_{\boldsymbol{b r}}=m_{r}\left[\begin{array}{c}
\boldsymbol{I}_{2 \times 2} \\
\mathbf{0}_{2\left(N_{R}-1\right) \times 2}
\end{array}\right] .
$$

It is clear from Eqs. (8) and (17) that for a perfect unsupported ring, the ring centre displacement only couples to the rigid body motion of the ring described via generalised coordinates $q_{1}^{(1)}$ and $q_{1}^{(2)}$.

\subsection{Ring strain energy}

The strain energy in a thin, in-extensible ring is given by [10]:

$$
U_{r}=\frac{E I}{2 r^{3}} \int_{0}^{2 \pi}\left(\frac{\partial^{2} w}{\partial \theta^{2}}+w\right)^{2} \mathrm{~d} \theta
$$

where $E I$ is the in-plane flexural rigidity of the ring.

Using the Ritz expansion of the radial component (Eqs. (2) to (4)) in this equation, it can be shown that the strain energy can be expressed as:

$$
U_{r}=\frac{1}{2} \boldsymbol{\Lambda}^{\mathrm{T}} \boldsymbol{K}_{r} \boldsymbol{\Lambda},
$$

where $\boldsymbol{K}_{\boldsymbol{r}}$ is a $2 N_{R} \times 2 N_{R}$ diagonal stiffness matrix whose $2 n$ 'th and (2n-1)'th entries are $E I \pi\left(n^{2}-1\right)^{2} / r^{3}$ i.e. the generalised ring stiffness for the $n \theta$-mode. For rigid body motions of the ring $(n=1)$, the ring stiffness is zero. 


\subsection{Support leg strain and kinetic energies}

It is assumed there are $N_{l}$ identical supporting legs uniformly spaced around the ring circumference and each support leg provides constant stiffness restoring forces in the radial and tangential directions and a point mass inertia. Numerical values for the equivalent stiffnesses in the radial $k_{r}$ and tangential $k_{t}$ directions, and inertia $m_{l}$ can be obtained using the finite element or alternative methods of analysis.

Using Eq. (2), the total strain energy in the support legs is given by:

$$
U_{l}=\sum_{j=1}^{N_{l}} U_{l}^{j}=\frac{1}{2} \boldsymbol{\Lambda}^{\mathrm{T}} \sum_{j=1}^{N_{l}} \boldsymbol{K}_{l}^{j} \boldsymbol{\Lambda}=\frac{1}{2} \boldsymbol{\Lambda}^{\mathrm{T}} \boldsymbol{K}_{l} \boldsymbol{\Lambda},
$$

where $U_{l}^{j}$ and $\boldsymbol{K}_{l}^{j}$ are the strain energy and stiffness matrix respectively for the $j$ 'th 125 support leg and:

$$
\boldsymbol{K}_{l}^{j}=\boldsymbol{\Psi}\left(\frac{2 j \pi}{N_{l}}+\alpha\right)\left[\begin{array}{cc}
k_{r} & 0 \\
0 & k_{t}
\end{array}\right] \boldsymbol{\Psi}^{\mathrm{T}}\left(\frac{2 j \pi}{N_{l}}+\alpha\right) .
$$

Angle $\alpha$ defines the angular position of the last leg (case $j=N_{l}$ ) relative to the reference frame used.

The properties of the total stiffness matrix $\boldsymbol{K}_{\boldsymbol{l}}$ depend on summing the terms in Eq. (12). It is shown in the Appendix that terms associated with the $n \theta$ and $p \theta$-modes 130 in the total stiffness matrix are not coupled provided that $(n \pm p) / N_{l} \neq$ integer. For all other cases non-zero terms are present and the non-zero terms depend on angle $\alpha$. A consequence of this is that the total stiffness matrix is diagonal if and only if $N_{R}<N_{l} / 2$. However, recalling that $N_{R}$ is the Ritz order of approximation, selecting $N_{R}<N_{l} / 2$ ignores high order mode coupling introduced by the support legs.

135 To demonstrate these properties, consider the case when $N_{l}$ is even and $N_{R}=N_{l} / 2$. In this case the total stiffness matrix can be expressed as follows:

$$
\boldsymbol{K}_{\boldsymbol{l}}=\left[\begin{array}{cccc}
\boldsymbol{K}_{\boldsymbol{l 1 , 1}} & & & \mathbf{0} \\
& \boldsymbol{K}_{l 2,2} & & \\
& & \ddots & \\
\mathbf{0} & & & \boldsymbol{K}_{l N_{l} / 2, N_{l} / 2}
\end{array}\right],
$$

where:

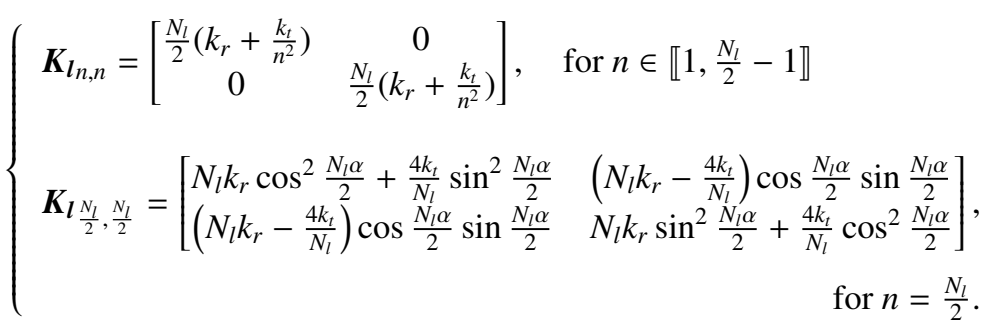

With the exception of the last two rows and columns, the matrix $\boldsymbol{K}_{\boldsymbol{l}}$ is diagonal with terms occurring in equal pairs and all terms independent of $\alpha$. In contrast the last two rows and columns, which correspond to the case when $n=N_{l} / 2$, depend 


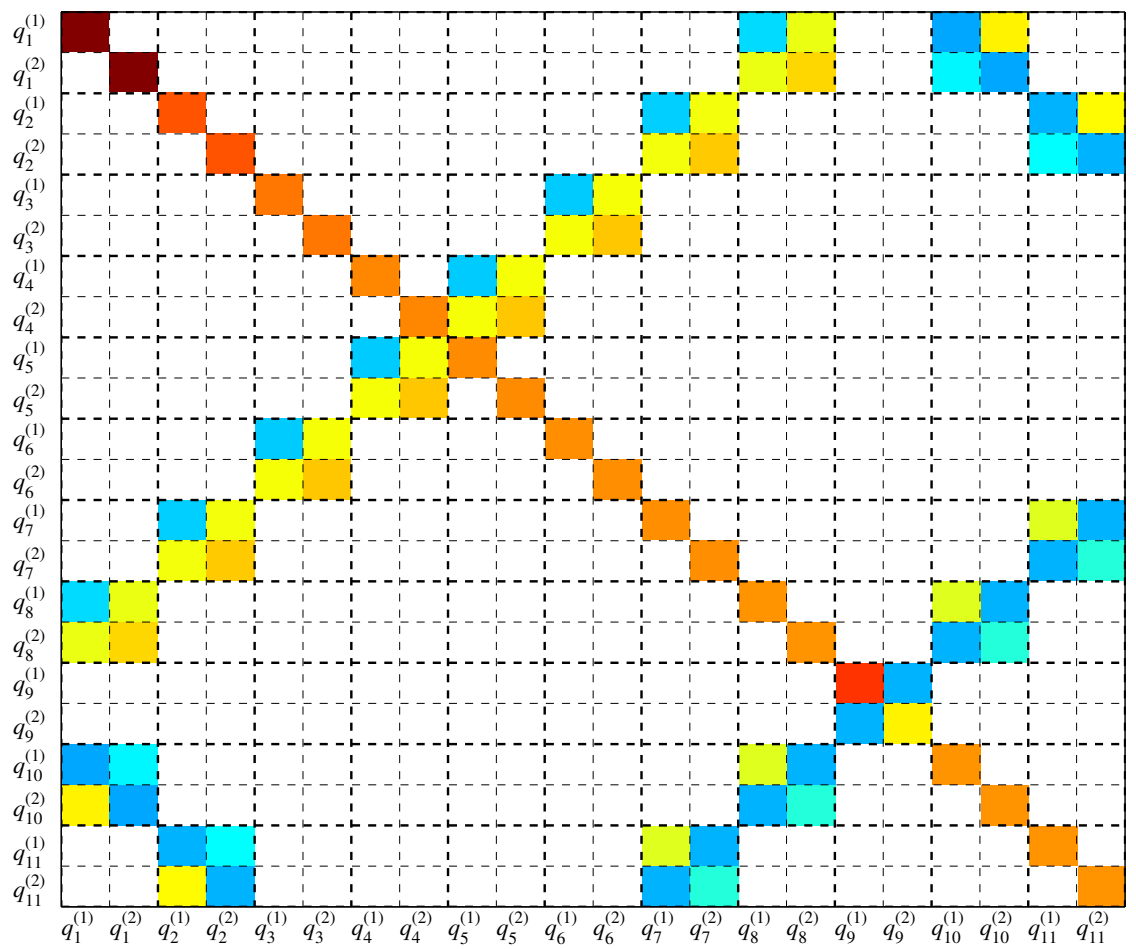

Figure 2: Illustration of stiffness matrix $\boldsymbol{K}_{l}$ showing the position of non-zero values, with $N_{l}=9$ and $N_{R}=11$

on $\alpha$ and have non-zero off-diagonal terms and unequal diagonal terms (even when $\alpha=0)$. A consequence of this is that the natural frequencies for the $n \theta$-modes of a ring with attached supports will split when $n=N_{l} / 2$. This is consistent with the so-called splitting rules [6,7] which indicate that frequency splits occur when $2 n / N_{l}$ is an integer. The vibrating gyroscope shown in Fig. 1 has eight supports $\left(N_{l}=8\right)$ and operates in the $2 \theta$-mode $(n=2)$, and so the number of supports does not split the $2 \theta$ frequencies. However, using eight supports will split the $4 \theta, 8 \theta, 12 \theta, \ldots$ modes.

An example illustrating when non-zero coupling terms occur in the total stiffness matrix is shown in Fig. 2 for the case when $N_{l}=9, N_{R}=11$ and $\alpha \neq 0$. Each cell represents the calculated value in the total stiffness matrix - white cells indicate zero values, shaded/coloured cells indicate non-zero values. The presence of coupling is clear. For example, the $(n=) 1 \theta$ and $(p=) 8 \theta$-modes, the $(n=) 4 \theta$ and $(p=) 5 \theta$ modes, and the $(n=) 2 \theta$ and $(p=) 11 \theta$-modes all couple because $(n \pm p) / N_{l}$ are integers. Along the main diagonal, the stiffness values occur in equal pairs, except when $n=9$ when the support legs induce frequency splitting. In practice the presence of coupling when $n$ is not equal to $p$ is expected to have a much smaller influence on frequency splitting than when $n$ is equal to $p$. This is because the frequencies for different modes of unsupported rings are normally well separated. For this reason, these terms are neglected in simulation studies performed later. 
Similar reasoning can be used to determine the overall mass matrix including support leg inertia. The total kinetic energy in the support legs is given by:

$$
T_{l}=\frac{1}{2} \dot{h}^{\mathrm{T}}\left[\begin{array}{cc}
\boldsymbol{M}_{\boldsymbol{l}} & \boldsymbol{M}_{\boldsymbol{b l}} \\
\boldsymbol{M}_{\boldsymbol{b l}}{ }^{\mathrm{T}} & N_{l} m_{l} \boldsymbol{I}_{2 \times 2}
\end{array}\right] \dot{\boldsymbol{h}},
$$

where $\boldsymbol{M}_{l}$ is a $2 N_{R} \times 2 N_{R}$ matrix having the same structure as $\boldsymbol{K}_{l}$ and whose first diagonal terms have the same form as Eq. (15) but with $k_{r}$ and $k_{t}$ replaced by $m_{l}$. These terms are simply equal to $N_{l} m_{l}\left(n^{2}+1\right) /\left(2 n^{2}\right)$ for $n<N_{l} / 2$. All of the $\boldsymbol{M}_{l}$ terms can

165 be found by using the same replacement in the Appendix. The coupling effect of the support masses on the kinetic energy is defined by:

$$
\boldsymbol{M}_{\boldsymbol{b l}}=N_{l} m_{l}\left[\begin{array}{c}
\boldsymbol{I}_{2 \times 2} \\
\mathbf{0}_{2\left(N_{R}-1\right) \times 2}
\end{array}\right] .
$$

\subsection{Electrostatic energy}

As the mean radius of the electrodes is normally large compared to the nominal capacitor gap size, the electrode capacitors are approximated as parallel plate capacitors. The voltages applied across the inner and outer capacitors are denoted $V_{i}$ and $V_{o}$ respectively, and the electrostatic energy stored by a differential element of the parallel plate capacitor is given by:

$$
\mathrm{d} E_{c}=\frac{\epsilon_{0} \epsilon V^{2}}{2 d} \mathrm{~d} A_{c},
$$

where $\epsilon$ is the relative permittivity of the ring material, $\epsilon_{0}$ is the absolute permittivity, $V$ is the electrical potential across the electrode-ring capacitor, $d$ is the gap size between 175 the capacitor and the ring, and $\mathrm{d} A C$ is the differential capacitor area. The gap size between the inner or outer capacitor and the ring depends of the nominal gap size (denoted $d_{g}$ ) and the radial displacement $w(\theta)$, such that:

$$
d=d_{g} \pm w(\theta) .
$$

Substituting Eq. (19) in Eq. (18) and expanding the denominator of the resulting equation as a Taylor series in terms of $w / d_{g}$ gives:

$$
\mathrm{d} E_{c}=\frac{\epsilon_{0} \epsilon V^{2}}{2 d_{g}} \sum_{k=0}^{N_{T}}(\mp 1)^{k}\left(\frac{w}{d_{g}}\right)^{k} \mathrm{~d} A_{c}
$$

where $N_{T}$ is the number of terms used in the Taylor series expansion.

The differential capacitor area $\mathrm{d} A c$ is defined as either $l r_{i} \mathrm{~d} \theta$ for the inner capacitor or $l r_{o} \mathrm{~d} \theta$ for the outer capacitor, where $r_{i}$ and $r_{0}$ are the inner and outer mean radii of the capacitor respectively.

Neighbouring electrodes are normally separated from each other by circumferential gaps to help isolate the electrodes and allow space for the support legs. These gaps are small compared to the span of the electrodes and can be neglected [5]. This assumption allows the electrodes to be merged together to form one continuous electrode around the complete ring circumference. The electrostatic energy stored by the inner or outer 
capacitors is obtained by integrating (20) around the circumference of the ring. The results in:

$$
E_{c}=A_{\epsilon} \sum_{k=0}^{N_{T}}\left(\frac{r_{o} V_{o}^{2}+(-1)^{k} r_{i} V_{i}^{2}}{d_{g}{ }^{k}} \int_{0}^{2 \pi} w^{k} \mathrm{~d} \theta\right)
$$

with

$$
A_{\epsilon}=\frac{\epsilon \epsilon_{0} l}{2 d_{g}}
$$

Using the Ritz expansion of the radial displacement (Eqs. (2) to (4)), Eq. (21) provides a polynomial expansion for the electrostatic energy in terms of generalised coordinates $q_{n}^{(1)}, q_{n}^{(2)}$ up to order $N_{T}$.

\subsection{Equation of motion}

Lagrange's equation is used to determine the equation of motion of the supported ring resonator:

$$
\frac{\mathrm{d}}{\mathrm{d} t}\left(\frac{\partial T}{\partial \dot{q}_{j}}\right)+\frac{\partial\left(U_{r}+U_{l}\right)}{\partial q_{j}}=\frac{\partial E_{c}}{\partial q_{j}} .
$$

Using the results developed earlier, the equation of motion can be written as:

$$
\left(M_{r}+M_{l}\right) \ddot{\Lambda}+\left(K_{r}+K_{l}+K_{c}\right) \Lambda+\bar{F}_{\mathrm{nl}}=F_{b}
$$

In this expression, $\boldsymbol{F}_{\boldsymbol{b}}=-\left(\boldsymbol{M}_{\boldsymbol{b} r}+\boldsymbol{M}_{\boldsymbol{b l}}\right) \ddot{z}_{b}$ represents the base excitation force applied to the central ring hub, indicating that for a perfect unsupported ring, the ring centre displacement resulting from an applied shock only couples to the rigid body motion of the ring. The first two entries of this vector are equal to $-\left(m_{r}+N_{l} m_{l}\right) \ddot{z}_{\boldsymbol{b}}$ and all other entries are zero. $\overline{\boldsymbol{F}}_{\mathrm{nl}}$ represents the nonlinear electrostatic force and is

205 a polynomial function of $\boldsymbol{\Lambda}$. This term arises from differentiating $E_{c}$ with respect to generalised coordinates $q_{n}^{(1)}, q_{n}^{(2)}$, i.e.:

$$
\overline{\boldsymbol{F}}_{\mathrm{nl}}=\left[\begin{array}{c}
\cdots \\
\cdots \\
-A_{\epsilon} \sum_{k=3}^{N_{T}} \frac{k\left(r_{o} V_{o}^{2}+(-1)^{k} r_{i} V_{i}^{2}\right)}{d_{g}{ }^{k}} \int_{0}^{2 \pi} w^{k-1} \cos j \theta \mathrm{d} \theta \\
-A_{\epsilon} \sum_{k=3}^{N_{T}} \frac{k\left(r_{o} V_{o}^{2}+(-1)^{k} r_{i} V_{i}^{2}\right)}{d_{g}{ }^{k}} \int_{0}^{2 \pi} w^{k-1} \sin j \theta \mathrm{d} \theta \\
\ldots \\
\cdots
\end{array}\right]
$$

The linear electrostatic stiffness terms have been separated from the nonlinear electrostatic terms and are incorporated in the linear stiffness matrix $\boldsymbol{K}_{\boldsymbol{c}}$. The linear terms are evaluated by setting $k=2$ in (21) to yield diagonal matrix:

$$
\boldsymbol{K}_{\boldsymbol{c}}=-2 \pi A_{\epsilon} \frac{r_{o} V_{o}^{2}+r_{i} V_{i}^{2}}{d_{g}{ }^{2}} \boldsymbol{I}
$$

210

The linear electrostatic forces provided by the outer and inner capacitors change the total stiffness of the system by applying a softening effect $\left(\boldsymbol{K}_{\boldsymbol{c}}\right.$ is a negative definite 
matrix). This softening effect increases as the electrode voltages increase, and the overall stiffness matrix of the system can become negative, making the system unstable, if the voltage is sufficiently large.

Some observations regarding the nonlinear electrostatic force are:

- If the electrode voltages are chosen such that $r_{o} V_{o}^{2}=r_{i} V_{i}^{2}$, all even power terms in the generalised coordinates (i.e. $k$ odd) in Eq. (25) cancel out, thereby eliminating some coupling mechanisms.

- Different terms in the nonlinear electrostatic force expansion exhibit softening or hardening behaviour (e.g. hardening occurs when $r_{i} V_{i}^{2}>r_{o} V_{o}^{2}$ ).

- The expression developed for the nonlinear electrostatic force can be expanded analytically, but becomes cumbersome as the number of terms in the Taylor series expansion $N_{T}$ increases and the Ritz order of approximation $N_{R}$ increases. Symbolic calculation software can be used without much difficulty to generate high order terms and evaluate the required integral terms.

Equation of motion (24) is rewritten by pre-multiplying by matrix $\left(\boldsymbol{M}_{\boldsymbol{r}}+\boldsymbol{M}_{l}\right)^{-1}$ and including a modal damping matrix $\boldsymbol{D}$ to give:

$$
\boldsymbol{I} \ddot{\boldsymbol{\Lambda}}+\boldsymbol{D} \dot{\boldsymbol{\Lambda}}+\boldsymbol{\Omega} \boldsymbol{\Lambda}+\boldsymbol{F}_{\mathrm{nl}}=\left[\begin{array}{c}
-\ddot{z_{b}} \\
\mathbf{0}
\end{array}\right]
$$

In this expression, matrix $\boldsymbol{\Omega}$ defines the undamped linear natural frequencies (including linear electrostatic effects) of the modes provided the stiffness $\left(\boldsymbol{K}_{l}\right)$ and mass ${ }_{230}\left(\boldsymbol{M}_{l}\right)$ matrices associated with the supports are diagonal - this occurs if the number of modes included in the model is relatively low $\left(N_{R}<N_{l} / 2\right)$ or the linear coupling is simply neglected because it is small. For the device shown in Fig. 1, which will be analyzed later, the stiffness and mass matrices contain coupling terms for the $4 \theta, 8 \theta$, $12 \theta, \ldots$ modes. However, this coupling can be avoided by selecting support leg angle

${ }_{235} \alpha=0$. Coupling also exists between different mode pairs, as discussed in the previous section (see Fig. 2). For example, coupling exists between $3 \theta$ and $5 \theta$-modes and this coupling can not be eliminated by selecting a particular value of $\alpha$. This coupling is neglected in the current study on the basis that it will be weak and to simplify the analysis. The damping matrix $\boldsymbol{D}$ is normally assumed to be diagonal and contains terms $\omega_{n} / Q_{n}$, where $Q_{n}$ is the quality factor (or $Q$-factor) and $\omega_{n}$ is the undamped natural frequency for each mode. $\boldsymbol{F}_{\mathrm{nl}}$ defines the influence of the nonlinear electrostatic forces including any coupling between generalised coordinates.

In summary, the equation of motion for the $n \theta$-mode can be expressed as:

$$
\ddot{q}_{n}^{(1)}+\frac{\omega_{n}}{Q_{n}} \dot{q}_{n}^{(1)}+\omega_{n}^{2} q_{n}^{(1)}+F_{\mathrm{nl}} q_{n}^{(1)}=F_{\mathrm{ext}_{q_{n}}^{(1)}}
$$

Similarly, the equation of motion for the companion $n \theta$ orthogonal mode is obtained by replacing $q_{n}^{(1)}$ by $q_{n}^{(2)}$ in (28). The external forces $F_{\text {ext }} q_{1}^{(1)}$ and $F_{\text {ext }} q_{1}^{(2)}$ correspond to the external base excitation forces applied to the ring and only excite the $1 \theta$-modes. Additional sources of excitation can be applied directly to the generalised coordinates. For example, harmonic forces are assumed to be applied to one of the $2 \theta$-modes in 
later studies by setting $F_{\text {ext } q_{2}^{(1)}}=\cos \omega t$ to replicate standard operating conditions for a Tibrating ring based gyroscope.

The nonlinear term $F_{\mathrm{nl}} q_{n}^{(1)}$ depends on the modal mass for the $n^{\text {th }}$ mode. For the purpose of simplicity, the contribution from support leg inertia on this nonlinear term has been neglected in later numerical simulations. This simplification will have a small influence on the amplitude of the nonlinear force but the general behaviour illustrated in Section 3 will be unaffected.

In Eq. (28), the electrostatic force provides the only source of nonlinearity. Under standard operating conditions, $\boldsymbol{F}_{\mathrm{nl}}$ can be neglected on the basis that the displacement of the ring is small compared to the gap size. However, in this study severe shock conditions are investigated which induce ring displacement in the order of the nominal 260 gap size, and it is necessary to model the nonlinear electrostatic forces. As described earlier, the nonlinearity is expressed in power series form and the level of approximation depends on the number of terms used in the Taylor expansion $N_{T}$. The polynomial order of the nonlinear force is $N_{T}-1$ and including these nonlinear forces introduces a physical mechanism for mode coupling to occur. Depending on the selected value of $N_{T}$, coupling can occur between several generalised coordinates.

\section{Shock simulations}

\subsection{Simulation data}

Numerical results are presented here for a recently reported device, manufactured and tested by Yoon et al. [1]. The dimensions of the ring are: mean radius $r=1.5 \mathrm{~mm}$, 270 radial thickness $h=18 \mu \mathrm{m}$, and axial length $l=150 \mu \mathrm{m}$. The nominal gap between capacitor and ring is $d_{g}=10 \mu \mathrm{m}$. The material properties are: Young's modulus $E=150 \mathrm{GPa}$ and density $\rho=2330 \mathrm{~kg} / \mathrm{m}^{3}$. The ring is supported by 8 pairs of legs. Using Eq. (28), the stiffnesses of the legs are accounted for within the natural frequencies $\omega_{n}$. These frequencies were obtained from a modal analysis of the resonator using a commercial Finite Element (FE) software package and the results are presented in Table 1. The electrostatic forces are quite week and in practice make only a small modification to the frequencies. On this basis it is justifiable to neglect their contribution to the linear frequencies. The natural frequency calculated for the $2 \theta$-mode (i.e. $\omega_{2}$ ) is in agreement with the results presented in [1]. The mode shapes obtained from 280 the FE analysis for $n \leq 3$ are shown in Fig. 3. Mode $n=1$ describes the $1 \theta$ ring rigid body motion, whilst the other modes $(n=2, n=3)$ describe the $2 \theta$ and $3 \theta$ flexural modes of the ring. The frequencies calculated for the $4 \theta$ and $5 \theta$-modes are provided. There are two $4 \theta$ frequencies because the eight support legs split the $4 \theta$ frequencies. In simulations, the high-frequency $4 \theta$-mode is used as it aligns with the support legs and satisfies the $\alpha=0$ condition. In practice, the modal analysis produces an assortment of leg dominated modes in addition to the $1 \theta$-mode, but these modes are not considered in the current model (28).

To simulate the dynamics of the gyroscope using Eq. (28), the damping for each mode is required and these are specified in (28) as $Q$-factors. The $Q$-factors used in 290 the simulations are presented in Table 1 and were obtained as explained below. The rigid body motion $Q$-value is often difficult to estimate because it depends on the bond 


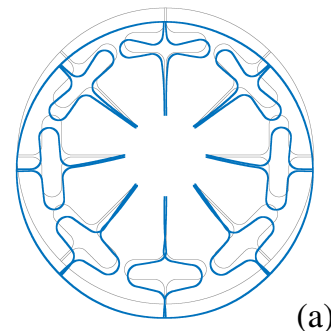

(a)

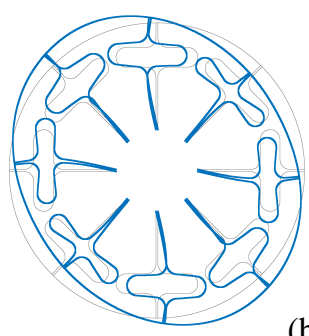

(b)

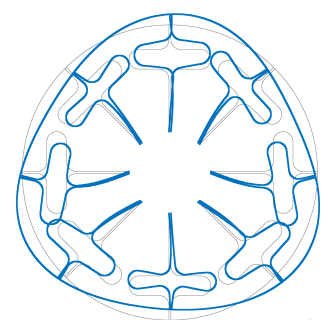

(c)

Figure 3: Undeformed shape and deformed mode shape of the ring gyroscope for (a) $n=1$, (b) $n=2$ and (c) $n=3$.

\begin{tabular}{c|c|c} 
mode number & Frequency $(\mathrm{kHz})$ & $Q$-factor \\
\hline $1 \theta$ & 11.2 & between 5000 and 180000 \\
\hline $2 \theta$ & 15.8 & 64700 from [1] \\
\hline $3 \theta$ & 22.0 & 170000 (estimated) \\
\hline $4 \theta$ & $25.7,36.3$ & 180000,80000 (estimated) \\
\hline $5 \theta$ & 59.2 & 50000 (estimated) \\
\hline
\end{tabular}

Table 1: Undamped natural frequencies and associated $Q$-factors for the first five ring modes.

connecting the central hub to the device and thermoelastic damping. In numerical simulations, a range of values of $Q_{1}$ is used. The $2 \theta$-mode $Q$-value was measured experimentally in [1] and the same value is kept within the simulations. The $Q$-values for the other flexural modes were not presented in [1] and so were calculated based on the assumption that thermoelastic damping is the only source of dissipation. The values presented in Table 1 were calculated using Zener's theory [11]. Using this approach it can be shown [12] that the $Q$-factor of each ring mode can be calculated using:

$$
\frac{1}{Q}=\frac{1}{Q_{r}} \frac{V_{r}}{V_{r}+V_{l}}+\frac{1}{Q_{l}} \frac{V_{l}}{V_{r}+V_{l}},
$$

where $Q_{r}$ and $Q_{l}$ are respectively the $Q$-factors for the ring and legs considered independently (see Eq. (30)), $V_{l}$ and $V_{r}$ are respectively the energy stored in the support legs and the ring for the mode considered - these quantities are obtain from an FE model of the undamped system. $Q_{r}$ and $Q_{l}$ are given by [12]:

$$
Q_{r, l}=\frac{1+\left(\tau_{r, l} \omega_{i}\right)^{2}}{\Delta_{M}}
$$

where $\omega_{i}$ is the fundamental undamped natural frequency of the supported ring for the mode considered, $\tau_{r, l}$ and $\Delta_{M}$ are respectively the effective relaxation time and relaxation strength defined as:

$$
\tau_{r, l}=\frac{C_{v} h_{r, l}^{2}}{k \pi^{2}} \quad \text { and } \quad \Delta_{M}=\frac{E \alpha^{2} T_{0}}{C_{v}} .
$$


Here $h_{r, l}$ is either the radial ring thickness $\left(h_{r}=18 \mu \mathrm{m}\right)$ or the leg width $\left(h_{l}=10 \mu \mathrm{m}\right)$; $C_{v}=\rho C_{p}$ (with $C_{p}=700 \mathrm{~J} \cdot \mathrm{kg}^{-1} \cdot \mathrm{K}^{-1}$ the specific heat for silicon) is the specific heat capacity at constant volume; $k=130 \mathrm{~W} \cdot \mathrm{m}^{-1} \cdot \mathrm{K}^{-1}$ is the thermal conductivity for silicon; $\alpha=2.6 \times 10^{-6} \mathrm{~K}^{-1}$ is the coefficient of thermal expansion for silicon; $T_{0}=300 \mathrm{~K}$ is the reference temperature.

The applied shock corresponds to an imposed acceleration of the base of the sensor and is accounted for in the definition of $\ddot{z}_{b}$ in Eq. (27). In the model it is represented as an applied external force on the $1 \theta$ rigid body mode and will be referred to as $F_{s}$ later. The applied shock is assumed to be a half-sine pulse [13] and is applied along the ${ }_{315} \alpha=0$ direction to eliminate any coupling in the stiffness matrix. As such, acceleration $\ddot{z_{b}}$ is expressed as:

$$
\ddot{z}_{b}=\left[\begin{array}{c}
F_{s} \\
0
\end{array}\right]=\left[\begin{array}{ll}
A_{s} \sin \frac{\pi t}{T_{s}} & \text { for } t \leq T_{s} \\
0 & \text { for } t>T_{s}
\end{array}\right],
$$

where $A_{s}$ and $T_{s}$ are respectively the amplitude and duration time of the applied shock. Throughout the following study, $T_{s}$ is chosen to be one tenth of the period of the $2 \theta$ mode. In severe shock conditions, the amplitude of the shock can be very high and for the device analyzed the maximum amplitude considered is $10000 \mathrm{~g}$ (a shock of $15000 \mathrm{~g}$ was applied by Yoon et al. in [1] but the shock duration was not mentioned) which causes the ring-electrode gap to reduce to approximately $60 \%$ of its nominal value. For higher levels of deformation the so-called "pull-in" phenomenon [14] is likely to occur, in which the ring would be pulled into contact with the electrode. The developed model and does not consider contact with the electrode.

The nonlinear coupling terms provide the only means of coupling the generalised coordinates. By aligning the external forcing with $\alpha=0$, there is no coupling with the orthogonal companion modes $q_{n}^{(2)}$. This halves the number of generalised coordinates that need to be included in the equations of motion, and in what follows only generalised coordinates $q_{n}^{(1)}$, corresponding to the $n \theta$-mode, are considered without their orthogonal companion. To simplify notation, superscript (1) will be removed and the generalised coordinates considered will be referred to as $q_{n}$.

Except when stated otherwise, the inner electrode is deactivated $\left(V_{i}=0\right)$ in below simulations.

\subsection{Preliminary study: influence of the nonlinear force order}

As the applied shock considered is severe $\left(A_{s}=10000 \mathrm{~g}\right)$, strong nonlinear effects are expected and the order of the Taylor series expansion (20) must be considered carefully to correctly predict these nonlinear effects. Initially a convergence study is performed to show the influence of using a different number of terms in the Taylor series expansion for the nonlinear electrostatic force.

Figure 4 shows the backbone curve for the nonlinear $1 \theta$-mode calculated using different values for the number of terms of $N_{T}$ in the Taylor expansion. A nonlinear mode is defined as a harmonic solution of the underlying autonomous conservative 345 system of Eq. (27):

$$
\boldsymbol{I} \ddot{\mathbf{\Lambda}}+\mathbf{\Omega} \boldsymbol{\Lambda}+\boldsymbol{F}_{\mathrm{nl}}=\mathbf{0}
$$


The Harmonic Balance Method (HBM) is employed to calculate the nonlinear mode (see [15] for a detailed explanation how to compute nonlinear modes). Non trivial solution of Eq. (33) are sought in the form of a Fourier ansatz:

$$
\boldsymbol{\Lambda}(t)=\boldsymbol{a}_{0}+\sum_{k=1}^{N_{h}}\left(\boldsymbol{a}_{k} \cos (k \omega t)+\boldsymbol{b}_{k} \sin (k \omega t)\right)
$$

where $N_{h}$ is the number of harmonics retained. Inserting Eq. (34) into (33) and evaluating Fourier-Galerkin projections with respect to the base functions gives rise to a system of nonlinear algebraic equations with unknowns $\boldsymbol{a}_{0}, \boldsymbol{a}_{k}, \boldsymbol{b}_{k}$ and $\omega\left(k \in \llbracket 1, N_{h} \rrbracket\right)$. To determine the nonlinear $1 \theta$-mode, this system of equations is solved by an arc-length continuation scheme [16] initiated with the linear frequency $\omega_{1}$ and a small value of $\boldsymbol{\Lambda}$.

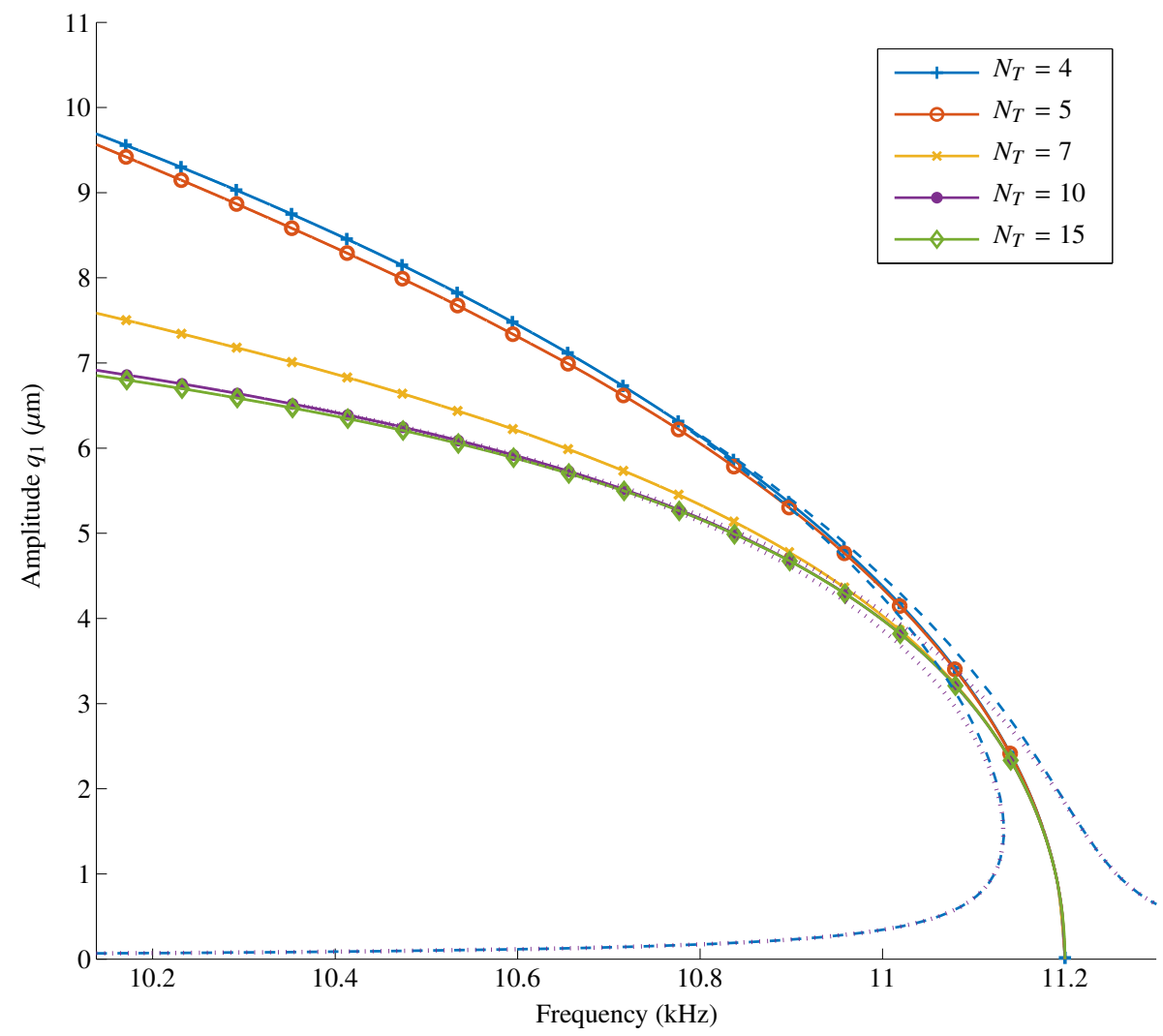

Figure 4: Nonlinear normal mode $q_{1}$ for different values of $N_{T}$

At low energy levels (low amplitude), the natural frequency of the nonlinear mode 355 is equal to the linear natural frequency $(11.2 \mathrm{kHz}$ for the $1 \theta$-mode). However as the energy in the system increases, or in our case as the $1 \theta$ vibration increases, the natural frequency decreases. This corresponds to a global softening effect induced by the nonlinear electrostatic forces. 
The advantage of studying nonlinear modes is that they show how the loci of the

frequency response functions (FRFs) plotted on top of the nonlinear modes. FRFs of the system for $N_{T}=4-$ dashed lines, and $N_{T}=10-$ dotted lines, are illustrated. These forced responses were obtained using arbitrary (but equal) forcing amplitudes to demonstrate that the bent peak follows the nonlinear modes. It is clear that de-

365 pending on the $N_{T}$-value, the response, be it forced or free, is different. Convergence for a maximum amplitude of approximately $6 \mu \mathrm{m}$ (corresponding to the $60 \%$ gap size displacement under applied severe shock) is obtained for $N_{T} \geq 10$.

In the following simulations, the number of terms in the Taylor series will be selected to be $N_{T}=10$, corresponding to a polynomial nonlinear force of order 9 .

\subsection{Free response after shock: influence of the number of modes}

In the first set of simulations, the resonator is considered to be at rest initially and is then subjected to a $10000 \mathrm{~g}$ shock on $q_{1}$ at $t=0$ as defined in Eq. (32). The amplitude of the shock is large and causes the ring-electrode gap to reduce to approximately $60 \%$ of its nominal value.

Figure 5 illustrates the radial displacement (defined in Eq. (2)) as a function of time for different Ritz orders of approximation $N_{R}$. For clarity, only the positive envelope of the response time-history is plotted to illustrate the global behaviour of the response over several seconds. The envelope was calculated by identifying local maxima in short duration windows of the multi-frequency time-history and then manually 380 combining and refining these maxima to achieve maxima points that encapsulate the global response behaviour. A sample showing the time response and positive envelope is illustrated as an embedded figure in Fig. 5 for a zoom on the $N_{R}=5$ simulation. The results in Fig. 5 show that the radial displacement envelope after shock is accurately simulated using the first 3 -modes of vibration (rigid body motion and $2 \theta$ and $3 \theta$ flexural modes). The main reason for this is that the modal amplitude decreases quickly as the mode number increases and the $q_{1}$ response dominates the response amplitude. For $N_{R}=5$, direct linear coupling between the $q_{5}$ and $q_{3}$ coordinates caused by the extra-diagonal terms in $\boldsymbol{K}_{\boldsymbol{l}}$ and $\boldsymbol{M}_{\boldsymbol{l}}$ matrices has been neglected.

Fig. 6 shows the positive envelope for modal amplitude $q_{5}$ calculated with $N_{R}=5$ together with a sample showing the time response and positive envelope for a zoom on the simulation. The results show a non-smooth behaviour and a rapid increase in amplitude at $t \approx 0.28 \mathrm{~s}$. To fully understand this singularity in the response, it is instructive to consider the evolution of the frequencies contained in the response over time. This is achieved by computing the spectrogram of the modal displacements. To calculate the spectrograms, the time-varying response is segmented into short periods and an FFT-based spectral estimate is performed over sliding windows [17]. The color and shades of the spectrograms encode frequency power levels. Dark color indicates frequency content with higher power, and a strong line (or ray) indicates for instance the existence of a particular frequency and shows its evolution over time. Figs. 7 and 8 show spectrograms for the $q_{1}$ and $q_{5}$ responses, respectively.

In Fig. 7, the spectrum of the response contains different rays, namely its fundamental natural frequency $\omega_{1}$ (around $11 \mathrm{kHz}$ ) and harmonics and combination of harmonics (for instance $\omega_{1}-\omega_{2}, 3 \omega_{1}, \omega_{1}+\omega_{2}$, etc.). The complexity of the spectrum is well 


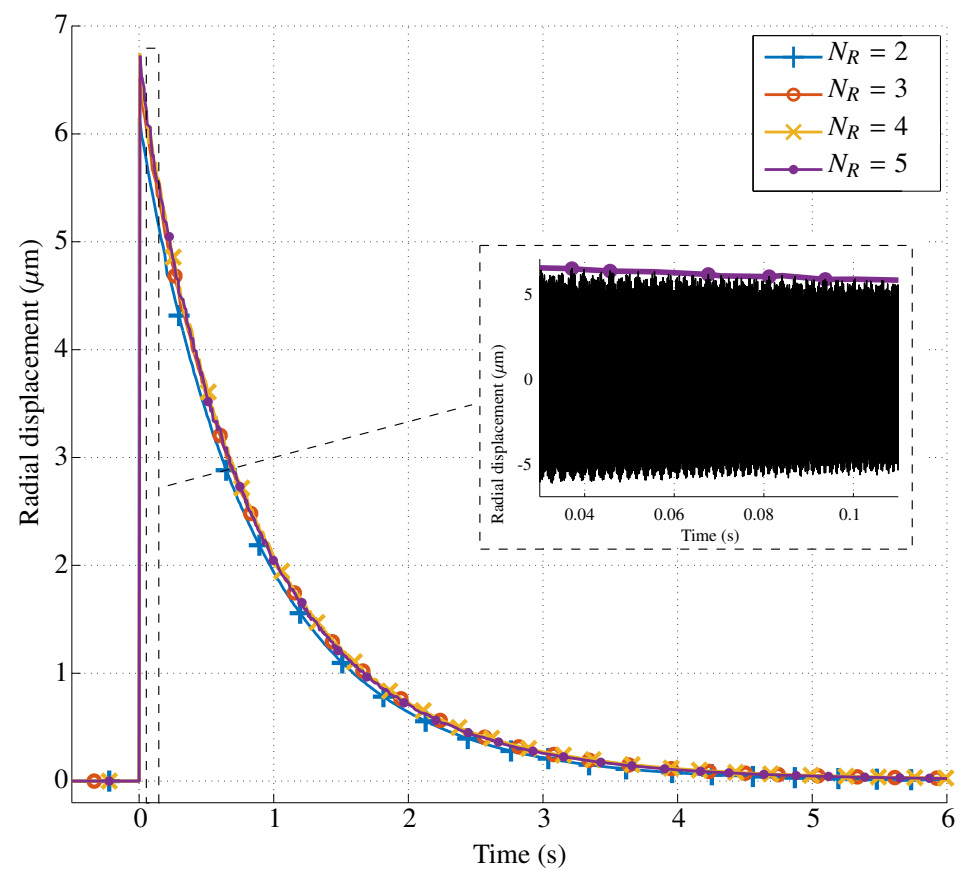

Figure 5: Shock simulations with structure initially at rest. Positive envelope of the radial displacement for different values of $N_{R}$.

known to be a consequence of nonlinear effects. The zoom shows how $\omega_{1}$ slowly increases over time from $10.6 \mathrm{kHz}$ to $11.2 \mathrm{kHz}$ as amplitude $q_{1}$ decays. This behavour is caused by the softening effect of the nonlinear forces which also explains the increase in frequency over time of combinations of harmonics.

Figure 8 shows even richer dynamics. In addition to the different harmonics or combination of harmonics, there is clear evidence of modal interaction at $t \approx 0.3 \mathrm{~s}$ and $\omega \approx 60 \mathrm{kHz}$. This modal interaction occurs when $\omega_{5}=2 \omega_{1}+\omega_{2}+\omega_{3}$ and creates an additional means of mode coupling. This energy exchange induces a peak in the $q_{5}$ response (see Fig. 6), but as the amplitude is relatively small compared to the amplitude of $q_{1}$, the peak is not visible in the global response of Fig. 5. Depending on the resonator dimensions, additional modal interactions are expected to occur.

\subsection{Forced harmonic response with applied shock}

Under operating conditions, one of the $2 \theta$-modes of a vibrating gyroscope is driven at resonance and the response of the companion $2 \theta$-mode is measured to provide a measure of the angular rate [18]. In this section the conditions when an applied shock significantly affects the forced vibration of the $2 \theta$-mode via inter-modal coupling are investigated when there is no applied rate.

A constant amplitude harmonic force is applied to the $2 \theta$-mode i.e. $F_{\text {ext }_{q_{2}}}=A_{e} \cos \omega t$ where $\omega$ is the excitation frequency. Figure 9 plots the amplitude of $q_{2}$ against excitation frequency $\omega$ near its linear resonant frequency. The excitation amplitude has been 


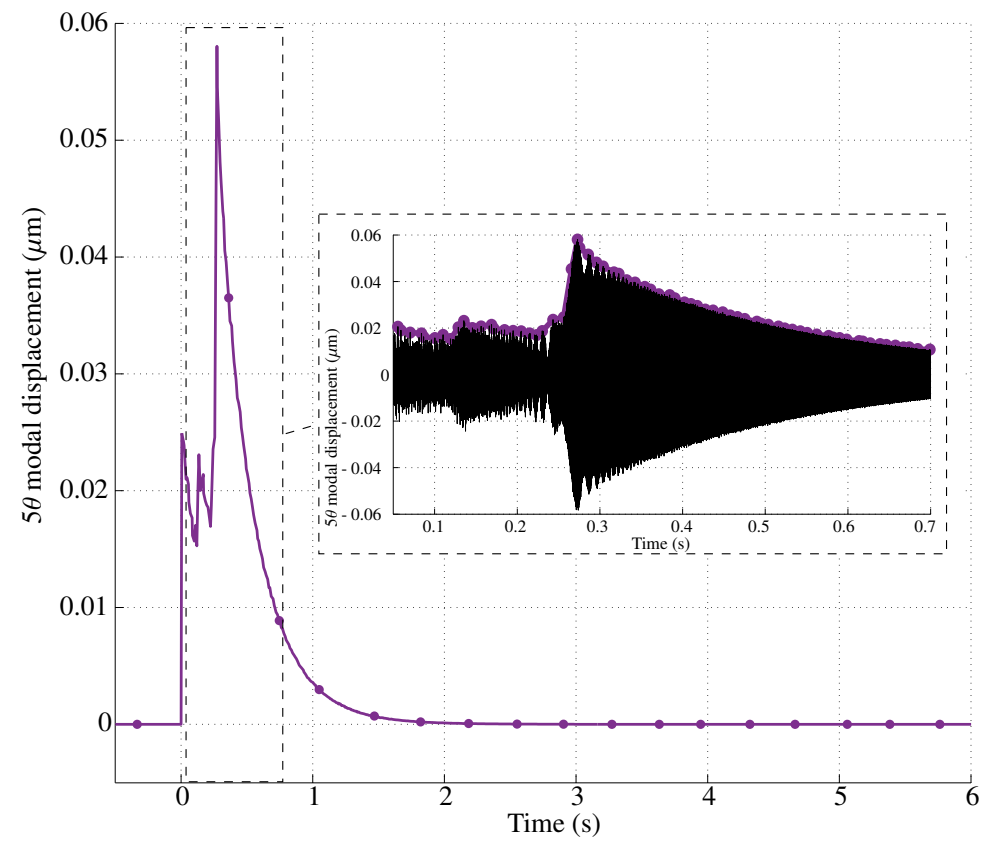

Figure 6: Shock simulations with structure initially at rest. Positive envelope of the modal displacement $q_{5}$ calculated with $N_{R}=5$.

chosen such that the drive amplitude $q_{2}$ is approximately $4 \%$ of the nominal gap size $(\approx 0.4 \mu \mathrm{m})$ and three modes $\left(N_{R}=3\right)$ are sufficient to characterize the global dynamics, see Section 3.3. In a way similar to the calculation of the nonlinear mode (see Section 3.2), the nonlinear response curve was found using a Harmonic Balance Method (HBM) that solves directly for the harmonic steady-state solutions, combined with an alternating frequency-time procedure (AFT) [19] to compute the projection of the non-

430 linear forces in the frequency domain, and arc-length continuation techniques [16] to follow a continuous branch of solution as the excitation frequency varies. Due to the presence of nonlinearity, the system presents two stable solutions over some frequency ranges, the top and bottom branches on Fig. 9. The results illustrate the softening behaviour of the nonlinear forces.

In the following simulation case studies, the $2 \theta$-mode only is driven at a frequency close to the peak of the nonlinear resonance (the actual frequency corresponds to the marker shown in Fig. 9, $w \approx 15.7983 \mathrm{kHz}$ ). Initially the system is maintained at its top stable branch solution $\left(q_{2} \approx 0.39 \mu \mathrm{m}\right)$. A shock is then applied to the $1 \theta$-mode, at $t=0 \mathrm{~s}$. The shock causes the ring to vibrate as a rigid body but also influences the 440 flexural vibration of the $2 \theta$-mode because of the coupling provided by the nonlinear electrostatic forces. Time-histories of the positive envelopes for the modal displacements are plotted in Figs. 10 to 13. The results presented are for particular cases using different values of shock excitation amplitude $\left(A_{s}\right)$ and $1 \theta$ damping $\left(Q_{1}\right)$. The details for the individual cases are as follows: 


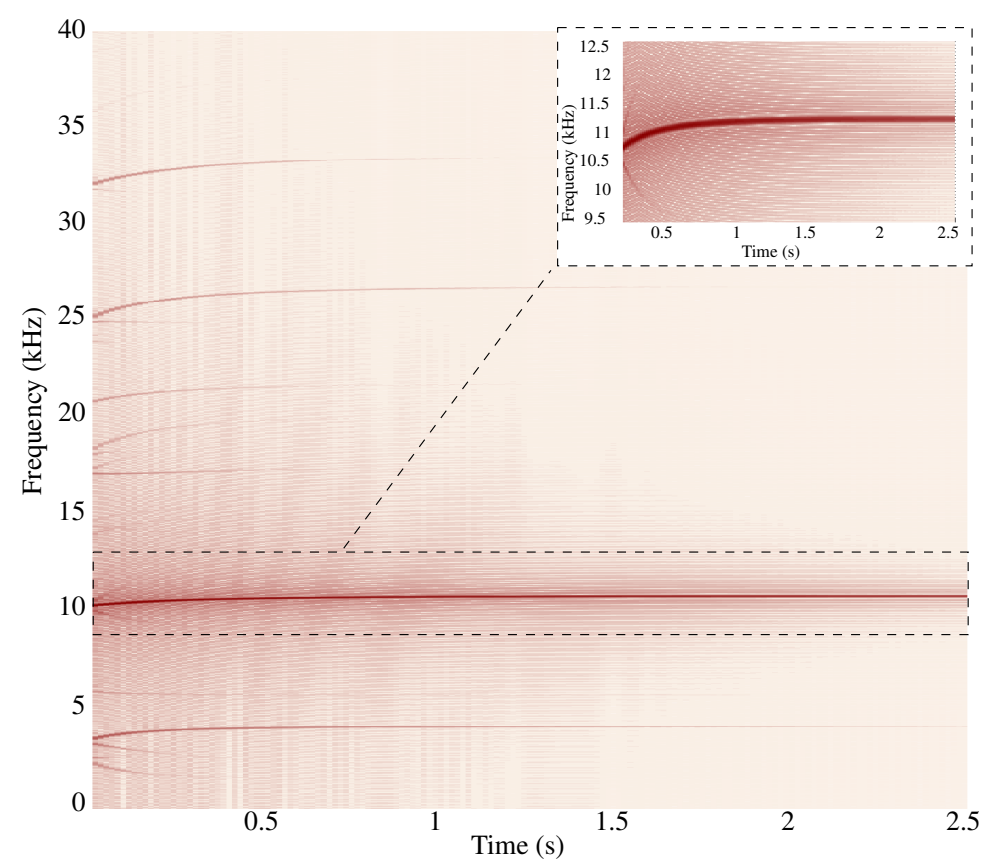

Figure 7: Shock simulations with structure initially at rest. Spectrogram of the $q_{1}$ response calculated with $N_{R}=5$.

Case 1. For this case the shock amplitude is relatively small $\left(A_{s}=2000 \mathrm{~g}\right)$ and $1 \theta$ damping low $\left(Q_{1}=120000\right)$, and the results are shown in Fig. 10. After a short transient response of approximately $7 \mathrm{~s}$, the system returns to its initial conditions where $q_{2}$ is at its top stable branch (round marker in Fig. 9). $q_{1}$ shows a slow exponential decay from damping.

Case 2. In this case the shock amplitude is large $\left(A_{s}=10000 \mathrm{~g}\right)$ and the damping low $\left(Q_{1}=120000\right)$. Due to the increased shock amplitude, which increases the ring deformation to $55 \%$ of its nominal gap size, the choice of $N_{T}$-value is important to achieve convergence, see Section 3.2. The results are shown in Fig. 11, where it can be seen that $q_{2}$ displays particularly interesting dynamic behaviour. After an initial transient response (up to approximately $5 s$ ), $q_{2}$ appears to stabilise on its bottom branch $q_{2} \approx 0.03 \mu \mathrm{m}$ (yellow cross marker of Fig. 9). However, while $q_{1}$ displacement slowly decays due to damping, $q_{2}$ returns to its initial condition on the top stable branch.

Case 3. In this case the shock amplitude is large $\left(A_{s}=10000 \mathrm{~g}\right.$ as in Case 2) but the damping on the $1 \theta$-mode is increased by a factor of $3\left(Q_{1}=40000\right)$. The results are shown in Fig. 12. It can be see that increasing the damping reduces the $q_{1}$ displacement more rapidly so it is negligible at around $7 \mathrm{~s}$. Also, after an initial transient 


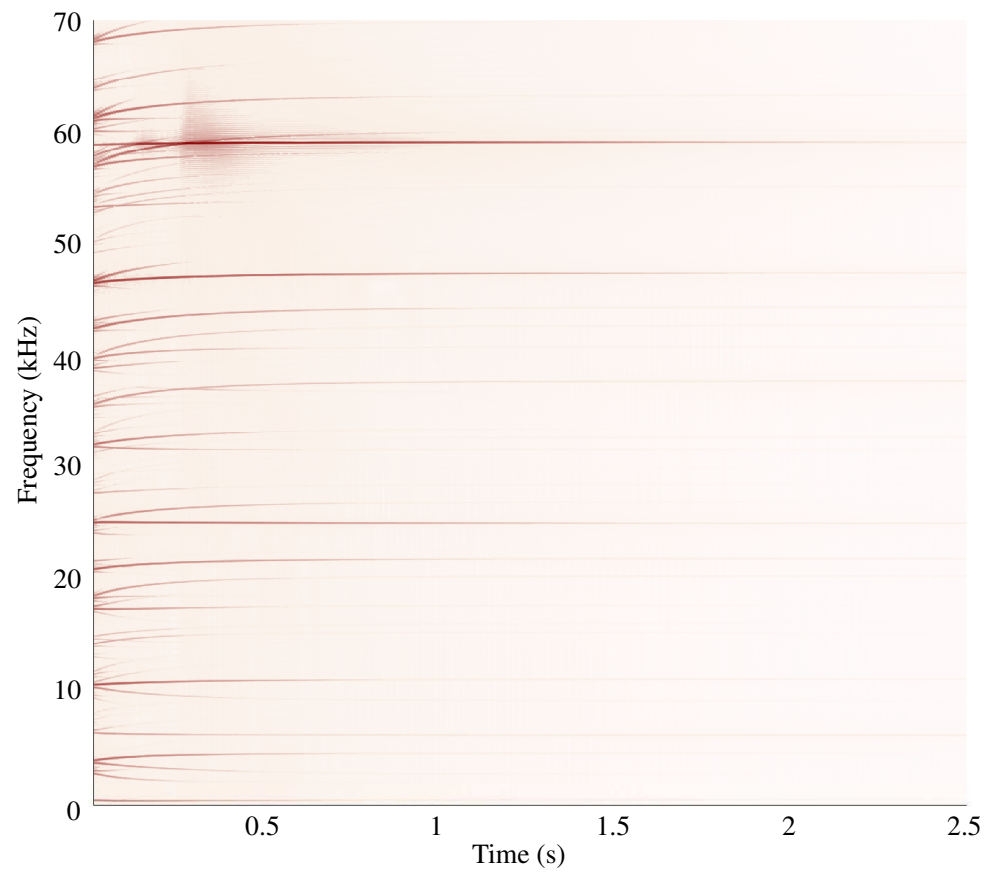

Figure 8: Shock simulations with structure initially at rest. Spectrogram of the $q_{5}$ response calculated with $N_{R}=5$.

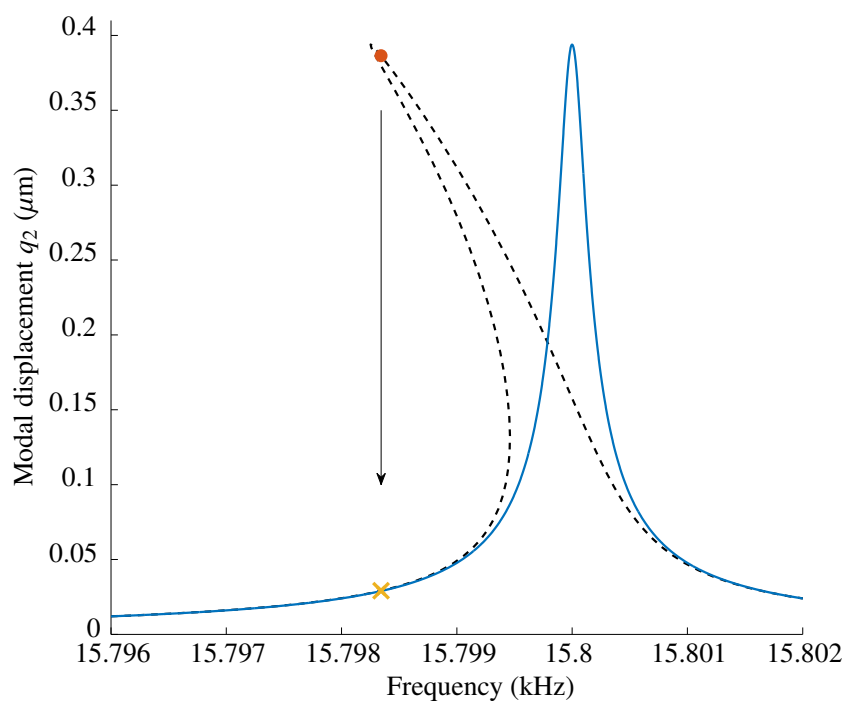

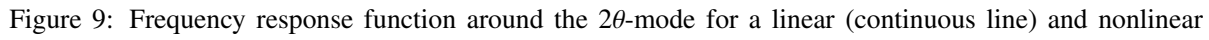
(dashed line) model. 


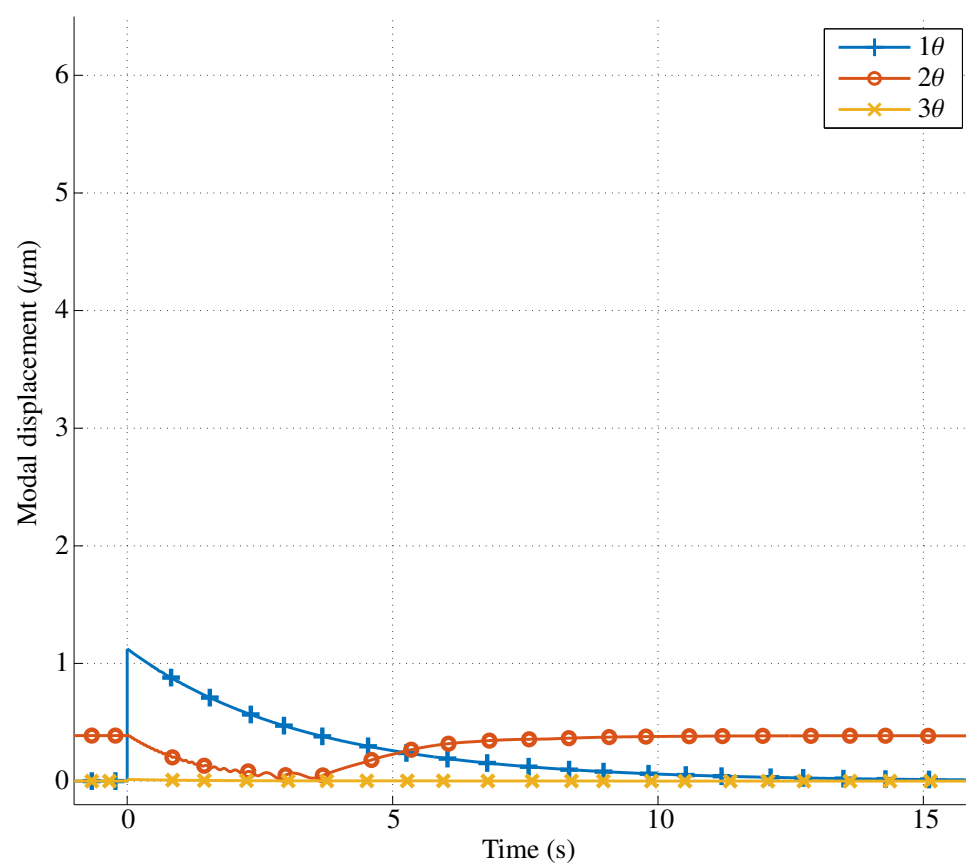

Figure 10: Positive envelope of the modal displacements as a function of time after a small shock - Case 1 .

$q_{2}$ stabilises and remains on the bottom stable branch. These results demonstrate the jump phenomenon from one branch to the other, as depicted by the arrow in Fig. 9.

Case 4. In this case the conditions necessary to cause the jump phenomenon observed in Case 3 are investigated by varying $Q_{1}$. As in Case 3, the shock amplitude is large but $Q_{1}$ is varied from 5000 to 180000 . The results for the $2 \theta$ response only are shown in Fig. 13. These results confirm the previous findings where the $q_{2}$ solution jumps to the bottom branch when the damping is high (low $Q_{1}$-value) but returns to the top branch after a sufficient stabilisation time when the damping is low (high $Q_{1}$-value).

The main conclusion drawn from this case studies is that for particular values of shock amplitude and damping the system exhibits jump behaviour from the high amplitude resonant driving state to a much lower amplitude. Even if the $2 \theta$ drive amplitude 475 is maintained initially, a shock applied to the rigid body $1 \theta$-mode can cause the ring amplitude to reduce suddenly. The jump phenomenon is caused by coupling between modes of vibration created by nonlinear effects that are normally not accounted for at the design stage. Furthermore, it has been shown that the abrupt jump down only occurs for high $1 \theta$ damping levels, which are often considered to be desirable as they quickly damp the rigid body motion. In practical applications, a control system may be used to maintain the $2 \theta$ drive response amplitude. In this case it is likely that the shock would reduce the drive response amplitude momentarily before eventually returning to 


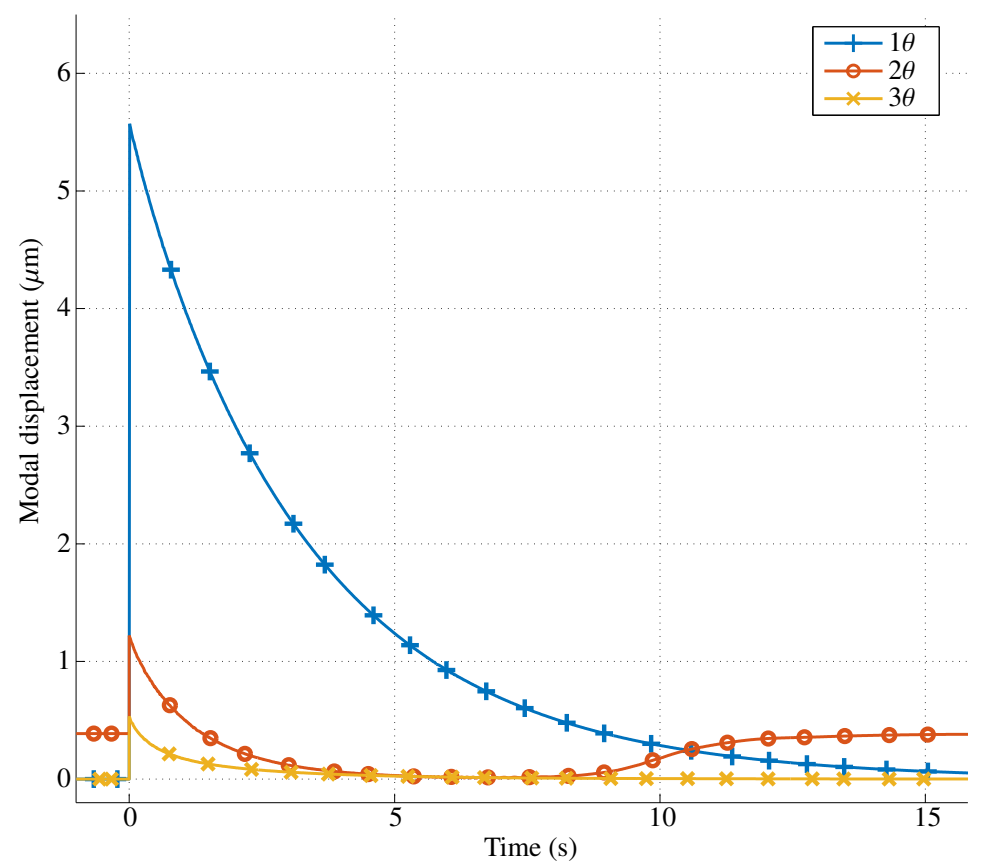

Figure 11: Positive envelope of the modal displacements as a function of time for a severe shock - Case 2.

the required steady-state amplitude.

\subsection{Influence of the inner electrode}

In all previous simulations, the inner voltage was set equal to 0 to simulate ring resonators with outer electrodes only. To gain some understanding of the benefits or otherwise of using inner as well as outer electrodes, it is necessary to reconsider the equations of motion. With the assumption that $N_{R}=2$, and $N_{T}=5$, the equations of motion (28) can be expressed as:

$$
\left\{\begin{array}{l}
\ddot{q}_{1}+\frac{\omega_{1}}{Q_{1}} \dot{q}_{1}+\omega_{1}^{2} q_{1}+\alpha q_{1} q_{2}-\beta q_{1}\left(q_{1}^{2}+2 q_{2}^{2}\right)+\gamma q_{1} q_{2}\left(4 q_{1}^{2}+3 q_{2}^{2}\right)=F_{s} \\
\ddot{q}_{2}+\frac{\omega_{2}}{Q_{2}} \dot{q}_{2}+\omega_{2}^{2} q_{2}+\frac{4 \alpha}{5} q_{1}^{2}-\frac{8}{5} \beta q_{2}\left(q_{2}^{2}+2 q_{1}^{2}\right)+\frac{4 \gamma}{5} q_{1}^{2}\left(2 q_{1}^{2}+9 q_{2}^{2}\right)=0
\end{array},\right.
$$

with:

$$
\alpha=\frac{3\left(r_{i} V_{i}^{2}-r_{o} V_{o}^{2}\right) A_{\epsilon} \pi}{\left(m_{r}+8 m_{l}\right) d_{g}{ }^{3}}, \quad \beta=\frac{3\left(r_{i} V_{i}^{2}+r_{o} V_{o}^{2}\right) A_{\epsilon} \pi}{\left(m_{r}+8 m_{l}\right) d_{g}{ }^{4}}, \quad \gamma=\frac{5 \alpha}{6 d_{g}^{2}}
$$

In this expression, there is no harmonic forcing applied to the $2 \theta$-mode.

As mentioned in Section 2.5, imposing the condition $r_{i} V_{i}^{2}=r_{o} V_{o}^{2}$ causes all of the even order terms in the nonlinear force expression to cancel out and eliminates some coupling mechanisms. Under these conditions the nonlinear force terms only include odd order terms and the above equation simplifies to:

$$
\left\{\begin{array}{l}
\ddot{q}_{1}+\frac{\omega_{1}}{Q_{1}} \dot{q}_{1}+\omega_{1}^{2} q_{1}-\beta q_{1}\left(q_{1}^{2}+2 q_{2}^{2}\right)=F_{s} \\
\ddot{q}_{2}+\frac{\omega_{2}}{Q_{2}} \dot{q}_{2}+\omega_{2}^{2} q_{2}-\frac{8}{5} \beta q_{2}\left(q_{2}^{2}+2 q_{1}^{2}\right)=0
\end{array}\right.
$$




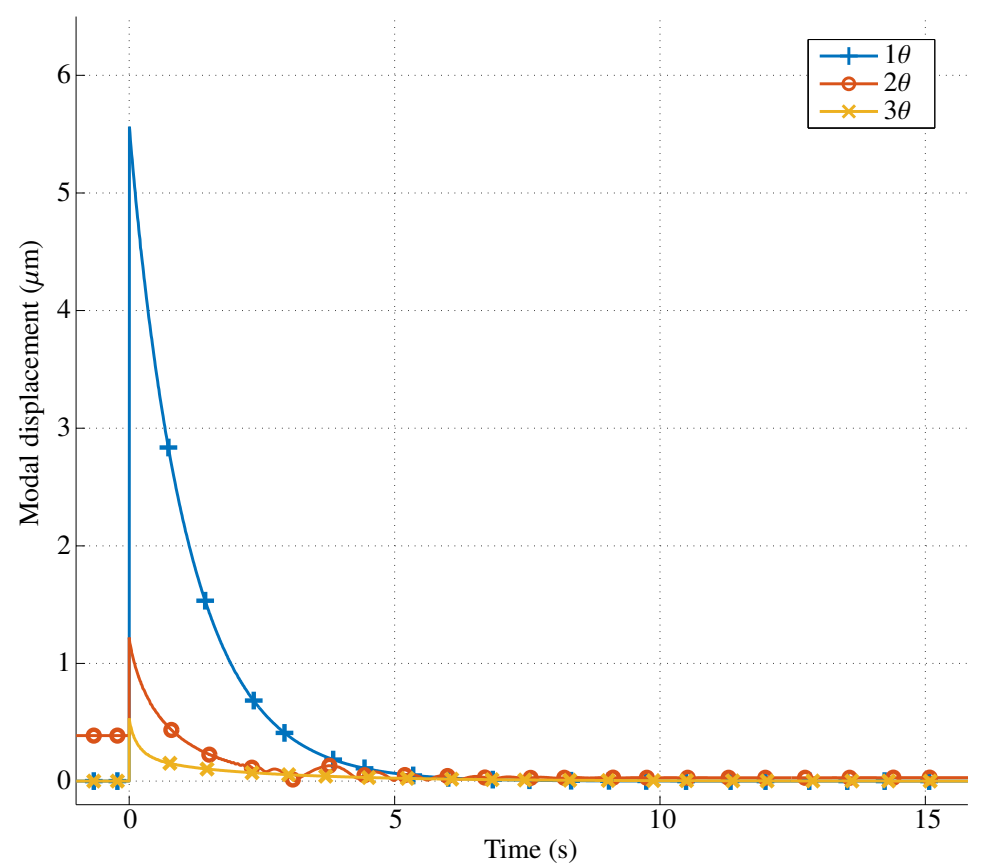

Figure 12: Positive envelope of the modal displacements as a function of time for a severe shock but with high damping - Case 3.

495 These equations indicate that if the system has zero initial conditions when the shock is applied, there is no coupling between $q_{1}$ and $q_{2}$ and $q_{2}=0$ is the solution to Eq. (36) for all values of $t$ and all $F_{s}$. This means that a shock applied to $q_{1}$ will not excite $q_{2}$. Simulation results for this case are shown in Fig. 14 and were obtained using $N_{R}=3$ and $N_{T}=10$. These results confirm that the applied shock does not produce any $q_{2}$ response for a more general form of equations. This phenomenon of "no coupling when the inner and outer voltages are symmetric" was previously mentioned in [4].

For the case when the initial conditions for $q_{2}$ are non-zero, like those considered in Section 3.4 where the $2 \theta$-mode is harmonically excited, the shock produces some coupling between generalised coordinates. Simulation results illustrating this behaviour 505 are shown in Fig. 15. The results show the positive envelopes of the modal displacements after the applied shock with a harmonic forcing applied to $q_{2}$. It can be seen that $q_{2}$ varies after the shock is applied and shows a jump behaviour similar to those illustrated in Fig. 13. A nonlinear modal interaction between $q_{1}$ and $q_{3}$ is also seen at approximately $t=0.1 \mathrm{~s}$ when there is a sudden increase in $q_{3}$ and decrease in $q_{1}$, indicating the transfer of vibrational energy from nonlinear coupling between $q_{1}$ and $q_{3}$. 


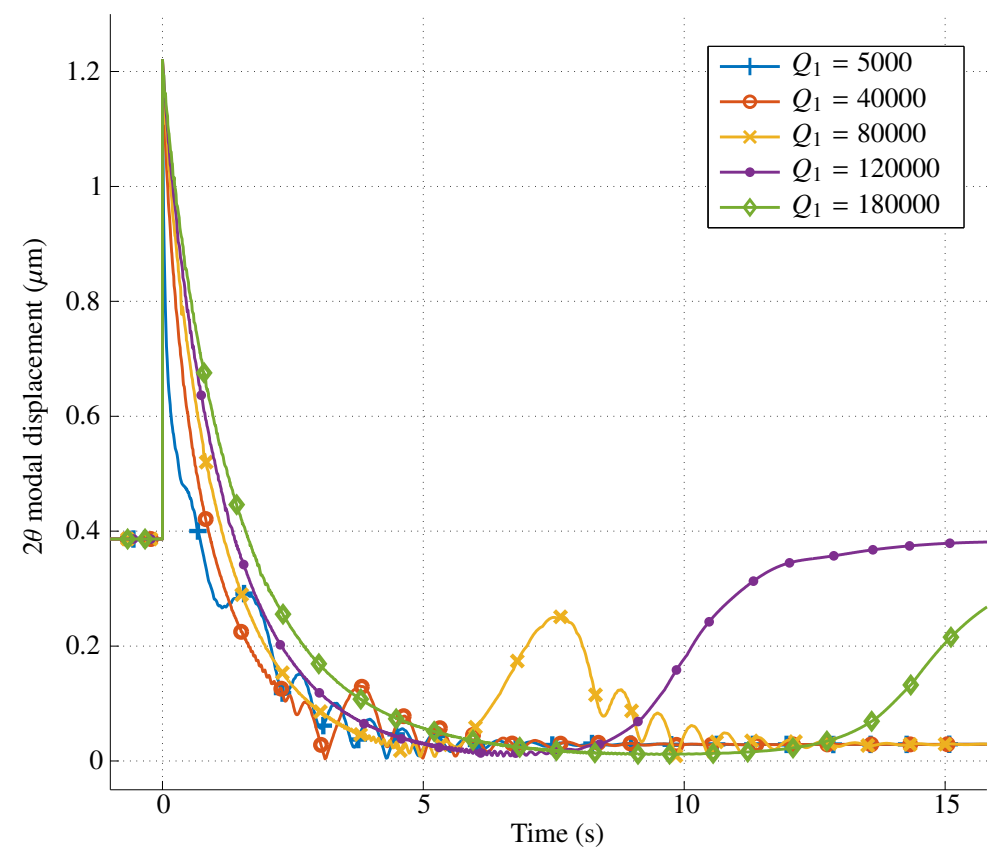

Figure 13: Positive envelope of the $q_{2}$ modal displacement as a function of time for a severe shock and different values of damping - Case 4.

\section{Conclusions}

A mathematical model has been developed and used to investigate the dynamic behaviour of ring resonators under severe shock conditions and nonlinear electrostatic forcing. The model describes the ring response in terms of the modes of a perfect ring, indicates that the nonlinear electrostatic forces induce mode coupling, and is used to simulate the resulting physical response.

The nonlinear electrostatic force was approximated using a Taylor series expansion and a study was performed to investigate the order of approximation required to reduces by $\sim 60 \%$, it is necessary to use a $9^{\text {th }}$ order approximation of the nonlinear electrostatic force and three modes of flexural vibration $(1 \theta, 2 \theta$ and $3 \theta)$ to achieve converged results for the global shock behaviour. Only three modes are required because the ring natural frequencies are well separated from each other; higher order modal responses decay quickly; and there is no linear coupling between the first ring modes. Electrostatic nonlinearity introduces coupling between the modes such that a shock which principally excites the rigid body mode produces flexural vibration of the ring and induces coupling between the $1 \theta$ and other modes. Under severe shock conditions sensor performance deteriorates compared to the linear case (without coupling). Fur530 thermore, under operating conditions when the $2 \theta$ mode is driven at resonance, it was demonstrated that it is possible for an applied shock to cause the vibrating response to jump to another stable state with much lower vibrating amplitude. The occurrence 


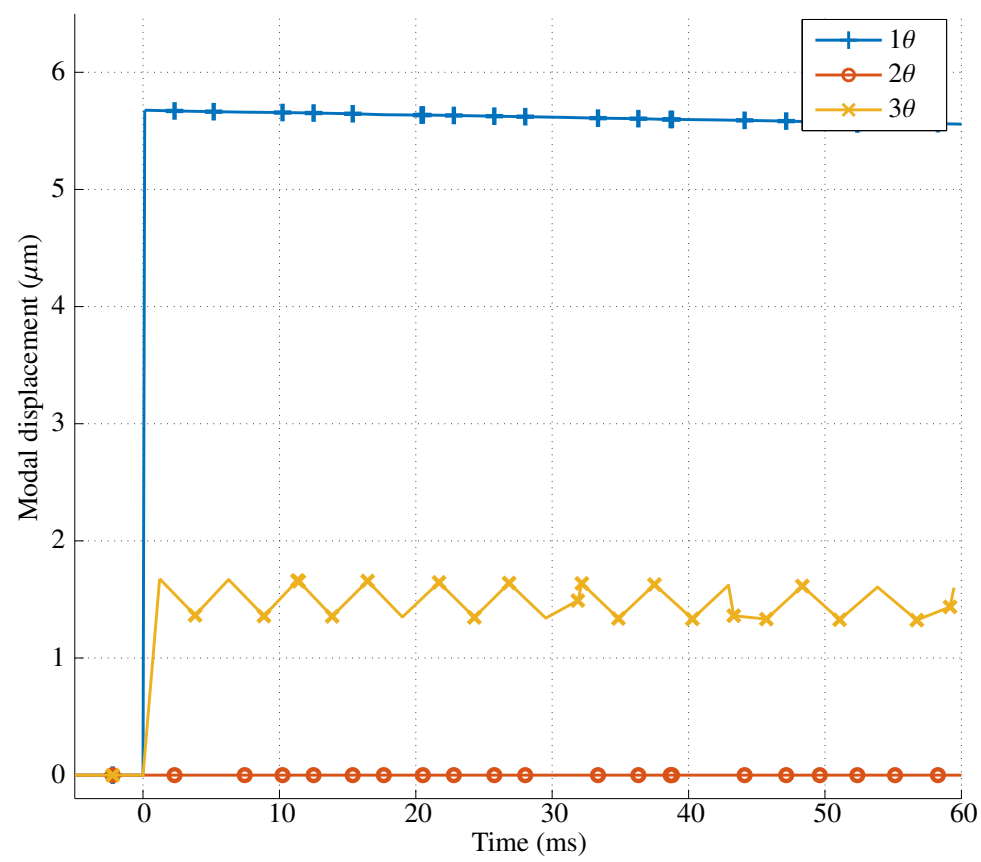

Figure 14: Positive envelopes of the modal displacements as a function of time for a severe shock without other external forces and with a symmetrical electrodes effect.

of this phenomenon is mainly a function of shock amplitude and rigid-body motion damping and is likely to reduce sensor performance momentarily. Finally, by incorporating both inner and outer electrodes the electrostatic restoring force can be made symmetric. This removes some coupling mechanisms between modes, but does not remove all couplings if the shock occurs when the resonator is vibrating.

A detailed analysis (bifurcation condition and stability studies) could be performed to more fully understand the complex dynamics created by the nonlinear electrostatic

540 force. Experimental measurements on CVG devices under severe shock conditions are also required to validate the findings and further investigate the conditions when the jump phenomenon occurs. It is anticipated that the model and results presented will guide future development of high performance capacitive CVGs.

\section{Acknowledgement}

The authors acknowledge the International Exchanges Scheme - Cost Share Programme - between the CNRS (PRC1088) in France and the Royal Society of London (IE150531) in the U.K. for funding this study. 


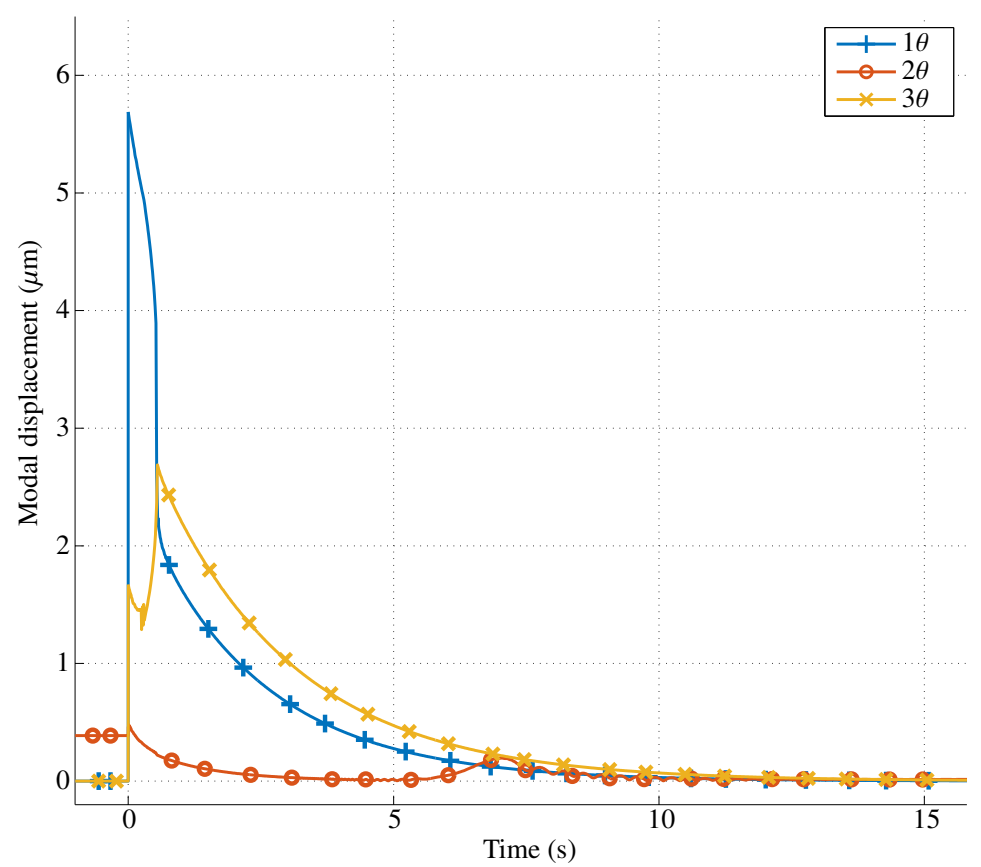

Figure 15: Positive envelope of the modal displacements as a function of time for a severe shock under harmonic forcing and with symmetrical electrodes effect.

\section{References}

[1] S. Yoon, U. Park, R. J., S. Yang, Tactical grade mems vibrating ring gyroscope with high shock reliability, Microelectronic Engineering 142 (2015) 22-29. doi : $10.1016 / j$. mee . 2015.07.004.

[2] A. D. Challoner, H. H. Ge, H. Y. Liu, Boeing disc resonator gyroscope, Position, Location and Navigation Symposium - PLANS 2014, IEEE/ION, Monterey, USA, 2014. doi:10.1109/PLANS . 2014.6851410.

[3] S. Yoon, S. Lee, K. Najafi, Vibration sensitivity analysis of mems vibratory ring gyroscopes, Sensors and Actuators, A: Physical 171 (2011) 163-177. doi:10. 1016/j.sna.2011.08.010.

[4] S. Sieberer, A. A. Popov, S. McWilliam, Shock-induced electrostatic coupling of modes of vibration in the response of a mems ring sensor, IDETC/CIE, Chicago, USA, 2012. doi:10.1115/DETC2012-70915.

[5] S. Sieberer, In-plane shock response of capacitive mems ring-rate senors, Ph.D. thesis, The University of Nottingham (2014).

[6] C. Fox, A simple theory for the analysis and correction of frequency splitting in slightly imperfect rings, Journal of Sound and Vibration 142 (3) (1990) 227-243. doi : 10.1016/0022-460X (90) 90554-D. 
[7] S. McWilliam, J. Ong, C. Fox, On the statistics of natural frequency splitting for rings with random mass imperfections, Journal of Sound and Vibration 279 (1-2) (2003) 453-470. doi:10.1016/j.jsv. 2003.11.034.

[8] F. Ayazi, K. Najafi, A HARPASS polysilicon vibrating gyroscope, Journal of Microelectromechanical Systems 10 (2001) 169-179. doi:10.1109/84.925732.

[9] Z. X. Hu, B. J. Gallacher, J. S. Burdess, C. P. Fell, K. Townsend, Precision mode matching of MEMS gyroscope by feedback control, Limerick, Ireland, 2011. doi:10.1109/ICSENS.2011.6126998.

[10] A. E. Love, A treatise on the mathematical theory of elasticity, $4^{\text {th }}$ Edition, Cambridge University Press, 2013.

[11] C. Zener, Internal friction in solids I. theory of internal friction in reeds, Physical Review 52 (3) (1937) 230-235. doi:10.1103/PhysRev .52.230.

[12] S. T. Hossain, Thermoelastic damping and support loss in MEMS ring resonators, Ph.D. thesis, The University of Nottingham, UK (2016).

[13] V. T. Srikar, S. D. Senturia, The reliability of microelectromechanical systems (mems) in shock environments, Journal of Microelectromechanical Systems 11 (3) (2002) 206-214. doi : 10 .1109/JMEMS . 2002 . 1007399.

[14] W.-M. Zhang, H. Yan, Z.-K. Peng, G. Meng, Electrostatic pull-in instability in mems/nems: A review, Sensors and Actuators, A: Physical 214 (2014) 187-218. doi:10.1016/j.sna.2014.04.025.

[15] M. Peeters, R. Viguié, G. Sérandour, G. Kerschen, J. C. Golinval, Nonlinear normal modes, part ii: Toward a practical computation using numerical continuation techniques, Mechanical Systems and Signal Processing 23 (1) (2009) 195-216. doi:10.1016/j.ymssp.2008.04.003.

[16] E. Sarrouy, J.-J. Sinou, Non-Linear Periodic and Quasi-Periodic Vibrations in Mechanical Systems - On the use of the Harmonic Balance Methods, Advances in Vibration Analysis Research, InTech, 2011, pp. 419-434. doi : 10.5772/15638.

[17] MATLAB, version 8.3.0 (R2014a), The MathWorks Inc., Natick, Massachusetts, USA, 2014.

595 [18] C. H. J. Fox, D. J. W. Hardie, Vibratory gyroscopic sensors, DGON Symposium Gyro Technology, Stuttgart, 1984, pp. 13.0-13.30.

[19] T. M. Cameron, J. H. Griffin, An alternating frequency/time domain method for calculating the steady-state response of nonlinear dynamic systems, Journal of Applied Mechanics 56 (1) (1989) 149-154. doi:10.1115/1.3176036. 


\section{Appendix}

$\boldsymbol{K}_{l}^{j}$ is composed of $\boldsymbol{K}_{l_{n, p}}^{j}$ matrices (size [2×2]) at rows $(2 n-1,2 n)$ and columns $(2 p-1,2 p)$, with $1<n<N_{R}$ and $1<p<N_{R}$.

$$
\boldsymbol{K}_{l}^{j}=\left[\begin{array}{lllll}
\ldots & \ldots & & \ldots & \ldots \\
\cdots & \ldots & & \ldots & \ldots \\
& & \boldsymbol{K}_{l n, p}^{j} & & \\
\ldots & \ldots & & \ldots & \ldots \\
\ldots & \ldots & & \ldots & \ldots
\end{array}\right]
$$

One introduces here an angle $\alpha$ that defines the position of the last leg with respect to the global frame. This angle can be arbitrarily taken. Substituting $\Psi\left(\frac{2 j \pi}{N_{l}}+\alpha\right)$ by its value (3) in (13) gives :

$$
\boldsymbol{K}_{l n, p}^{j}=\left[\begin{array}{ll}
k_{r} a_{c c}^{j}+\frac{k_{t}}{n p} a_{s s}^{j} & k_{r} a_{c s}^{j}-\frac{k_{t}}{n p} a_{s c}^{j} \\
k_{r} a_{s c}^{j}-\frac{k_{t}}{n p} a_{c s}^{j} & k_{r} a_{s s}^{j}+\frac{k_{t}}{n p} a_{c c}^{j}
\end{array}\right]
$$

with :

$$
\begin{aligned}
& a_{c c}^{j}=\cos \left(\frac{2 n j \pi}{N_{l}}+n \alpha\right) \cos \left(\frac{2 p j \pi}{N_{l}}+p \alpha\right) \\
& a_{s s}^{j}=\sin \left(\frac{2 n j \pi}{N_{l}}+n \alpha\right) \sin \left(\frac{2 p j \pi}{N_{l}}+p \alpha\right) \\
& a_{c s}^{j}=\cos \left(\frac{2 n j \pi}{N_{l}}+n \alpha\right) \sin \left(\frac{2 p j \pi}{N_{l}}+p \alpha\right) \\
& a_{s c}^{j}=\sin \left(\frac{2 n j \pi}{N_{l}}+n \alpha\right) \cos \left(\frac{2 p j \pi}{N_{l}}+p \alpha\right)
\end{aligned}
$$

In order to calculate the total strain energy (12), one needs to calculate $\sum_{j=1}^{N_{l}} a_{j}^{c c}$, $\sum_{j=1}^{N_{l}} a_{j}^{s s}, \sum_{j=1}^{N_{l}} a_{j}^{c s}$ and $\sum_{j=1}^{N_{l}} a_{j}^{s c}$. This derivation follows.

$$
\begin{aligned}
\sum_{j=1}^{N_{l}} a_{j}^{c c} & =\sum_{j=1}^{N_{l}} \cos \left(\frac{2 n j \pi}{N_{l}}+n \alpha\right) \cos \left(\frac{2 p j \pi}{N_{l}}+p \alpha\right) \\
& =\frac{1}{2} \sum_{j=1}^{N_{l}}\left[\cos \left(\frac{2(n+p) j \pi}{N_{l}}+(n+p) \alpha\right)+\cos \left(\frac{2(n-p) j \pi}{N_{l}}+(n-p) \alpha\right)\right]
\end{aligned}
$$

Utilising the fact that:

$$
\sum_{j=1}^{N_{l}} \cos \left(\frac{2 n j \pi}{N_{l}}+n \alpha\right)= \begin{cases}N_{l} \cos n \alpha & \text { if } n / N_{l} \in \mathbb{Z} \\ 0 & \text { otherwise }\end{cases}
$$


Equation (43) gives :

$$
\begin{aligned}
\sum_{j=1}^{N_{l}} a_{j}^{c c} & = \\
& \begin{cases}\frac{N_{l}}{2} \cos (n-p) \alpha & \text { if }(n-p) / N_{l} \in \mathbb{Z} \text { and }(n+p) / N_{l} \notin \mathbb{Z} \\
\frac{N_{l}}{2} \cos (n+p) \alpha & \text { if }(n-p) / N_{l} \notin \mathbb{Z} \text { and }(n+p) / N_{l} \in \mathbb{Z} \\
N_{l} \cos n \alpha \cos p \alpha & \text { if }(n-p) / N_{l} \in \mathbb{Z} \text { and }(n+p) / N_{l} \in \mathbb{Z} \\
0 & \text { otherwise. }\end{cases}
\end{aligned}
$$

A similar reasoning can be performed for $\sum_{j=1}^{N_{l}} a_{j}^{s s}, \sum_{j=1}^{N_{l}} a_{j}^{c s}$, and $\sum_{j=1}^{N_{l}} a_{j}^{s c}$. The value of each sum depends of some conditions on the row and column number ( $n$ and $p$ respectively). These are summaries below.

\begin{tabular}{c|c|c|c|c}
$\sum$ & \multicolumn{4}{|c}{ Condition on $p$ and $n$} \\
\hline & $\begin{array}{c}(n-p) / N_{l} \in \mathbb{Z} \\
\text { and }(n+p) / N_{l} \notin \mathbb{Z}\end{array}$ & $\begin{array}{c}(n-p) / N_{l} \notin \mathbb{Z} \\
\text { and }(n+p) / N_{l} \in \mathbb{Z}\end{array}$ & $\begin{array}{c}(n-p) / N_{l} \in \mathbb{Z} \\
\text { and }(n+p) / N_{l} \in \mathbb{Z}\end{array}$ & otherwise \\
\hline position in $\boldsymbol{K}_{l}$ & positive diag. & negative diag. & intersection of diag. & everywhere else \\
\hline$\sum_{j=1}^{N_{l}} a_{j}^{c c}$ & $\frac{N_{l}}{2} \cos (n-p) \alpha$ & $\frac{N_{l}}{2} \cos (n+p) \alpha$ & $N \cos n \alpha \cos p \alpha$ & 0 \\
$\sum_{j=1}^{N_{l}} a_{j}^{s s}$ & $\frac{N_{l}}{2} \cos (n-p) \alpha$ & $-\frac{N_{l}}{2} \cos (n+p) \alpha$ & $N \sin n \alpha \sin p \alpha$ & 0 \\
$\sum_{j=1}^{N_{l}} a_{j}^{c s}$ & $-\frac{N_{l}}{2} \sin (n-p) \alpha$ & $\frac{N_{l}}{2} \sin (n+p) \alpha$ & $N \cos n \alpha \sin p \alpha$ & 0 \\
$\sum_{j=1}^{N_{l}} a_{j}^{s c}$ & $\frac{N_{l}}{2} \sin (n-p) \alpha$ & $\frac{N_{l}}{2} \sin (n+p) \alpha$ & $N \sin n \alpha \cos p \alpha$ & 0
\end{tabular}

